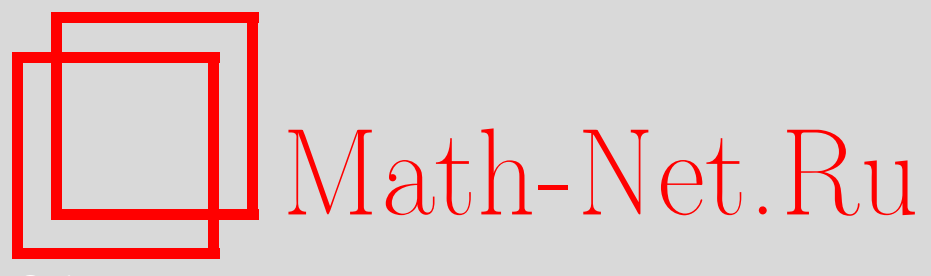

О. К. Шейнман, Алгебры операторов Лакса и гамильтоновы интегрируемые иерархии, УМН, 2011, том 66, выпуск 1, 151-178

DOI: https://doi.org/10.4213/rm9406

Использование Общероссийского математического портала Math-Net.Ru подразумевает, что вы прочитали и согласны с пользовательским соглашением http://www . mathnet.ru/rus/agreement

Параметры загрузки:

IP : 3.81 .55 .215

26 апреля 2023 г., 10:03:42

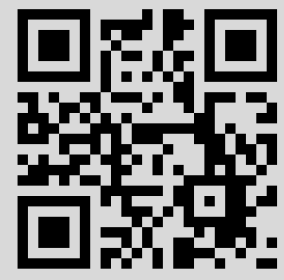




\title{
Алгебры операторов Лакса и гамильтоновы интегрируемые иерархии
}

\author{
О. К. Шейнман
}

\begin{abstract}
Мы рассматриваем теорию лаксовых уравнений со спектральным параметром на римановой поверхности, предложенную И. М. Кричевером в 2001 г. Наш подход базируется на новом объекте - алгебрах операторов Лакса, и обобщает подход И. М. Кричевера, вводя в него произвольную комплексную простую или редуктивную классическую алгебру Ли. Для каждого оператора Лакса, рассматриваемого как отображение, сопоставляющее точке кокасательного расслоения на расширенном пространстве данных Тюрина элемент соответствующей алгебры операторов Лакса, мы строим иерархию попарно коммутирующих потоков, заданную уравнениями Лакса, и доказываем, что они гамильтоновы относительно симплектической структуры Кричевера-Фонга. Соответствующие гамильтонианы задают интегрируемые конечномерные системы типа систем Хитчина. В качестве примера мы выводим в рамках нашего подхода эллиптические системы Калоджеро-Мозера типов $A_{n}, C_{n}, D_{n}$.

Библиография: 13 названий.
\end{abstract}

Ключевые слова: бесконечномерные алгебры Ли, алгебры токов, лаксовы интегрируемые системы, гамильтонова теория.

\section{СОДЕРЖАНИЕ}

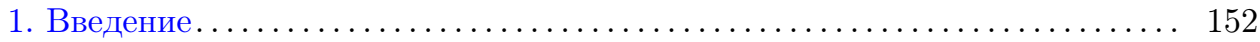

2. М-операторы ........................................ 154

3. $L$-операторы и алгебры операторов Лакса .................... 158

4. g-значные уравнения Лакса ............................. 160

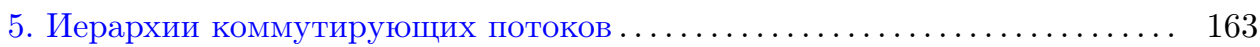

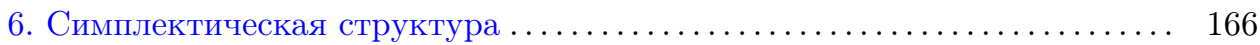

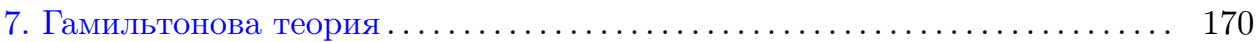

8. Примеры: системы Калоджеро-Мозера ..................... 173

Список литературы ................................... 177

Работа выполнена при поддержке РФФИ (грант № 08-01-00054-а) и программы “Фундаментальные проблемы нелинейной динамики" Президиума РАН.

(C) О.К. ШЕйнман, 2011 


\section{1. Введение}

В [1], [2] И. М. Кричевер и С. П. Новиков предложили метод нахождения конечнозонных решений ранга больше единицы для уравнений КадомцеваПетвиашвили и Шрёдингера. Основываясь на идеях этой работы и на своих результатах по эффективной классификации пар коммутирующих дифференциальных операторов ранга больше единицы [3], И. М. Кричевер предложил теорию операторов Лакса со спектральным параметром на римановой поверхности [4]. В [5] И. М. Кричевер и автор показали, что такие операторы Лакса образуют ассоциативную алгебру, и построили их ортогональные и симплектические аналоги, которые образуют уже алгебры Ли. Последние были названы алгебрами операторов Лакса. Алгебры операторов Лакса образуют новый класс одномерных алгебр токов.

Приложения алгебр токов к теории лаксовых уравнений имеют долгую историю. Они инициированы в работах И. М. Гельфанда, Л. А. Дикого, И. Я. Дорфман, А. Г. Реймана, М. А. Семенова-Тян-Шанского, В. Г. Дринфельда, В. В. Соколова, В. Г. Каца, П. ван Мёрбеке. Эти приложения связаны главным образом с алгебрами Каца-Муди, которые естественно возникают в контексте лаксовых уравнений с рациональным спектральным параметром. В [4] теория общепринятых лаксовых представлений и представлений нулевой кривизны с рациональным спектральным параметром была обобщена на случай алгебраической кривой $\Sigma$ произвольного рода $g$. Такие представления возникают в теории интегрируемых систем несколькими способами - например, в [1] введено представление нулевой кривизны уравнения Кричевера-Новикова, а в [4] построен теоретико-полевой аналог системы Калоджеро-Мозера на эллиптической кривой. Алгебры операторов Лакса возникают как соответствующее обобщение алгебр Каца-Муди.

В [6], [7] мы поставили задачу обобщения теории Кричевера [4] на все алгебры операторов Лакса и сделали первые шаги в этом направлении, включая построение интегрируемых иерархий лаксовых уравнений. В настоящей работе мы предпринимаем заключительный шаг: доказываем, что эти лаксовы уравнения гамильтоновы, и строим соответствующие гамильтонианы. Мы рассматриваем в контексте нашего подхода конкретные хорошо известные примеры (эллиптические системы Калоджеро-Мозера).

Понятие оператора Лакса на алгебраической кривой тесно связано с результатами А.Н. Тюрина по классификации голоморфных векторных расслоений на алгебраических кривых [8]. Оно использует данные Тюрина, моделирующие параметры Тюрина таких расслоений. Данные Тюрина состоят из точек $\gamma_{s} \in \Sigma(s=1, \ldots, n g)$ и ассоциированных с ними элементов $\alpha_{s} \in \mathbb{C} P^{n}$, где $g$ обозначает род римановой поверхности $\Sigma$, а $n$ соответствует рангу расслоения. Каждая алгебра операторов Лакса ассоциирована с фиксированными данными Тюрина и набором отмеченных точек на $\Sigma$. Для конечномерной простой или редуктивной алгебры Ли $\mathfrak{g}$ над $\mathbb{C}$ мы обозначаем через $\overline{\mathfrak{g}}$ соответствующую алгебру операторов Лакса. Это почти градуированная алгебра Ли. Ее элементы параметризованы кокасательными векторами к пространству данных Тюрина в соответствующей точке. Они введены в [4], [5] как мероморфные 
$(n \times n)$-матричнозначные функции на $\Sigma$, имеющие произвольные полюсы в точках $P_{k}, k=1, \ldots, N$ (которые предполагаются фиксированными) и полюсы порядка не более двух в точках $\gamma_{s}$. Коэффициенты лорановских разложений этих матричнозначных функций в окрестности точки $\gamma_{s}$ должны удовлетворять некоторым ограничениям, параметризованным векторами $\alpha_{s}$ (соотношения (3.4) ниже). В случае, когда точки $\gamma_{s}$ отсутствуют (что соответствует тривиальному векторному расслоению), мы возвращаемся к известному классу алгебр Кричевера-Новикова (см. обзор в [9]). Если вдобавок род $\Sigma$ равен 0 , $\sharp\left\{P_{i}\right\}=2$ и эти две точки суть 0 и $\infty$, мы получаем алгебры петель. Заметим здесь, что все эти три типа алгебр токов имеют сходную теорию центральных расширений [5], [10].

Под оператором Лакса мы понимаем отображение, сопоставляющее точке кокасательного расслоения на пространстве данных Тюрина (расширенных отмеченными точками) элемент соответствующей алгебры операторов Лакса. С каждым положительным дивизором $D=\sum m_{i} P_{i}$ мы связываем подпространство $\mathscr{L}^{D}$ кокасательного расслоения такое, что оператор Лакса из этого подпространства, как функция на $\Sigma$, имеет в $P_{i}$ полюс порядка не более $m_{i}$ для каждой точки $P_{i}$. Мы рассматриваем на $\mathscr{L}^{D}$ динамическую систему, заданную уравнением Лакса, и доказываем, что такие динамические системы гамильтоновы и интегрируемы по отношению к симплектической структуре Кричевера-Фонга.

В разделе 2, следуя [4], [7], мы вводим $M$-операторы (партнеры операторов Лакса в лаксовых парах) и изучаем их аналитические и алгебраические свойства. Каждый $M$-оператор определяет поток в кокасательном расслоении на пространстве расширенных данных Тюрина. Соответствующая динамика параметров Тюрина (2.7) играет фундаментальную роль. Она возникла в [1] как анзац, позволяющий эффективно проинтегрировать уравнение КадомцеваПетвиашвили в классе решений ранга выше 1, она также существенно используется в [4].

В разделе 3 мы даем краткий обзор алгебр операторов Лакса. Следуя [4], [7], мы вводим их элементы ( $L$-операторы $)$ как $M$-операторы, задающие тривиальную динамику данных Тюрина. Существует и независимое определение $L$-операторов, данное в [5]. Мы отсылаем к этой статье за подробностями.

В разделе 4 мы доказываем, что уравнение Лакса эквивалентно системе уравнений на главные части оператора Лакса в точках $P_{i}$ в совокупности с вышеупомянутыми уравнениями движения данных Тюрина. Мы доказываем условие корректной определенности динамической системы, задаваемой уравнением Лакса на пространстве $\mathscr{L}^{D}$.

В разделе 5 мы строим иерархии коммутирующих потоков на кокасательном расслоении на $\mathscr{L}^{D}$, заданные оператором Лакса. Ключевую роль играет лемма 5.1, в которой говорится о размерности пространства $M$-операторов, удовлетворяющих условиям раздела 4. Во всем кроме этой леммы мы следуем работе [4]. Мы получаем окончательный результат для всех рассматриваемых классических алгебр Ли, в частности для $\mathfrak{s p}(2 n)$, что не было сделано в более ранней работе автора по иерархиям [7]. Мы подчеркиваем, что $L$ и $M$, вообще 
говоря, не принадлежат одной и той же алгебре Ли, и приводим список соответствующих пар алгебр Ли (4.2). Заметим, что метод построения иерархий, предложенный в этом разделе, не является единственно возможным. В разделе 8 мы даем для эллиптических кривых другую его версию, позволяющую включить в рамки нашего подхода системы Калоджеро-Мозера.

В разделах 6, 7 мы развиваем гамильтонову теорию лаксовых уравнений рассматриваемого типа. В разделе 6 мы строим аналог симплектической структуры Кричевера-Фонга на кокасательном расслоении на некотором пространстве $\mathscr{P}^{D} \subset \mathscr{L}^{D} / G($ где $\mathfrak{g}=\operatorname{Lie}(G))$, инвариантном относительно потоков нашей коммутирующей иерархии. В разделе 7 мы показываем, что иерархии раздела 5 гамильтоновы по отношению к этой структуре, и строим соответствующие гамильтонианы. Изложение опять-таки аналогично для всех рассматриваемых классических алгебр Ли и, за исключением доказательства невырожденности формы Кричевера-Фонга (раздел 6) и голоморфности спектров операторов лаксовой пары (раздел 7), следует работе [4]. Это также делается отдельно для каждого класса алгебр Ли. Мы, кроме того, даем более подробные доказательства предложений работы [4].

В разделе 8 мы рассматриваем в рамках нашего подхода три известных примера интегрируемых систем. Первый связан с эллиптической системой Калоджеро-Мозера для $\mathfrak{g}=\mathfrak{g l}(n)$. Он был ранее рассмотрен в [4], [11]. Далее, модифицируя технику раздела 5 , мы получаем лаксовы операторы и соответствующие гамильтоновы интегрируемые иерархии для эллиптических систем Калоджеро-Мозера, связанных с $\mathfrak{s o}(2 n)$ и $\mathfrak{s p}(2 n)$. K сожалению, мы не знаем явной формы оператора Лакса нашего типа для системы, отвечающей $\mathfrak{s o}(2 n+1)$. Заметим, что в [12] лаксовы представления со спектральным параметром на эллиптической кривой получены для всех неприводимых приведенных систем корней. В них используются операторы Лакса, являющиеся функциями типа Бейкера-Ахиезера, в отличие от наших операторов Лакса, являющихся мероморфными функциями. Тем не менее, эти два типа лаксовых представлений калибровочно эквивалентны, подробнее см. в разделе 8.

Эта работа посвящена И. М. Кричеверу в честь его шестидесятилетия. Именно И. М. Кричеверу я обязан своим интересом к интегрируемым системам. Я глубоко признателен ему за многочисленные беседы на эту и другие темы, в том числе и за обсуждение этой статьи. Этой работой я отдаю дань восхищения его достижениям.

Я приношу свою благодарность М. Шлихенмайеру, а также А. Г. Сергееву и участникам его семинара в Математическом институте им. В.А. Стеклова за обсуждение некоторых аспектов этой работы и содержательные замечания.

\section{2. $M$-операторы}

Пусть $\Sigma$ - компактная риманова поверхность рода $g$ с двумя отмеченными точками $P_{+}, P_{-}$и $\mathfrak{g}-$ алгебра Ли над $\mathbb{C}$ из следующего списка: $\mathfrak{g l}(n), \mathfrak{s l}(n)$, $\mathfrak{s o}(2 n), \mathfrak{s o}(2 n+1), \mathfrak{s p}(2 n), \mathfrak{s}(n), \mathfrak{t} \mathfrak{p}(2 n)$, где $\mathfrak{s}(n)$ - алгебра скалярных матриц, a $\mathfrak{t} \mathfrak{p}(2 n)$ - подалгебра алгебры Ли $\mathfrak{s p}(2 n+2)$, состоящая из матриц с нулевыми первым столбцом и последней строкой. Это список из предыдущей работы [7], 
расширенный алгеброй $\mathfrak{t} \mathfrak{s}(2 n)$, которая возникает в конструкции интегрируемых иерархий в симплектическом случае.

Зафиксируем дополнительно $K$ точек $\gamma_{s} \in \Sigma$, и пусть

$$
W:=\left\{\gamma_{s} \in \Sigma \backslash\left\{P_{+}, P_{-}\right\} \mid s=1, \ldots, K\right\}
$$

(значение $K$ будет уточнено в разделе 5 ). Каждой точке $\gamma_{s}$ сопоставим вектор $\alpha_{s} \in \mathbb{C}^{p}$, заданный с точностью до скалярного множителя, где $p$ - размерность стандартного ("векторного") представления соответствующей алгебры Ли $\mathfrak{g}$ (т. е. элементы $\mathfrak{g}$ - это $(p \times p)$-матрицы). Система данных

$$
T:=\left\{\left(\gamma_{s}, \alpha_{s}\right) \mid s=1, \ldots, K\right\}
$$

называется ниже даннъми Тюрина. Эти данные связаны с пространством модулей голоморфных векторных расслоений на $\Sigma$. В частности, для $\left(\gamma_{s}, \alpha_{s}\right)$ общего положения, $\alpha_{s} \neq 0$ и $K=n g$ данные Тюрина параметризуют полустабильные оснащенные голоморфные расслоения на $\Sigma$ ранга $n$ и степени $n g$ [8]. Допуская вольность обозначений, будем опускать нижний индекс $s$ и писать просто $\{(\gamma, \alpha)\}$ вместо $\left\{\left(\gamma_{s}, \alpha_{s}\right) \mid s=1, \ldots, K\right\}$.

Пусть $M: \Sigma \rightarrow \mathfrak{g}-$ мероморфная функция. Мы требуем, чтобы в точке $\gamma=\gamma_{s}$ она имела разложение

$$
M=\frac{M_{-2}}{\left(z-z_{\gamma}\right)^{2}}+\frac{M_{-1}}{z-z_{\gamma}}+M_{0}+\cdots,
$$

где $z$ - фиксированная локальная координата в окрестности $\gamma, z_{\gamma}$ - координата самой точки $\gamma$, коэффициенты $M_{-2}, M_{-1}, M_{0}, M_{1}, \ldots$ принадлежат $\mathfrak{g}$ и

$$
M_{-2}=\lambda \alpha \alpha^{t} \sigma, \quad M_{-1}=\left(\alpha \mu^{t}+\varepsilon \mu \alpha^{t}\right) \sigma,
$$

где $\lambda \in \mathbb{C}, \mu \in \mathbb{C}^{n}, \sigma$ - матрица размера $n \times n$, а $t$ вверху обозначает транспонирование матрицы,

$$
\begin{aligned}
\lambda \equiv 0, & \varepsilon=0, & \sigma=\mathrm{id} & \text { для } \mathfrak{g}=\mathfrak{g l}(n), \mathfrak{s l}(n), \\
\lambda \equiv 0, & \varepsilon=-1, & \sigma=\mathrm{id} & \text { для } \mathfrak{g}=\mathfrak{s o}(n), \\
& \varepsilon=1 & & \text { для } \mathfrak{g}=\mathfrak{s p}(2 n)
\end{aligned}
$$

и в последнем случае $\sigma$ - матрица симплектической формы. В случае $\mathfrak{g}=$ $\mathfrak{t s p}(2 n)$ мы требуем, чтобы $\alpha$ и $\mu$ имели вид $\alpha=\left(\alpha_{0}, \widetilde{\alpha}^{t}, 0\right)^{t}\left(\alpha_{0} \in \mathbb{C}, \widetilde{\alpha} \in \mathbb{C}^{2 n}\right)$, $\mu=\left(\mu_{0}, \widetilde{\mu}^{t}, 0\right)^{t} \quad\left(\mu_{0} \in \mathbb{C}, \widetilde{\mu} \in \mathbb{C}^{2 n}\right)$, а $\sigma$ имела вид $\sigma=\left(\begin{array}{ccc}0 & 0 & 1 \\ 0 & \widetilde{\sigma} & 0 \\ -1 & 0 & 0\end{array}\right)$, где $\widetilde{\sigma}-$ матрица симплектической формы, определяющей $\mathfrak{s p}(2 n)$. Для такой матрицы $\sigma$

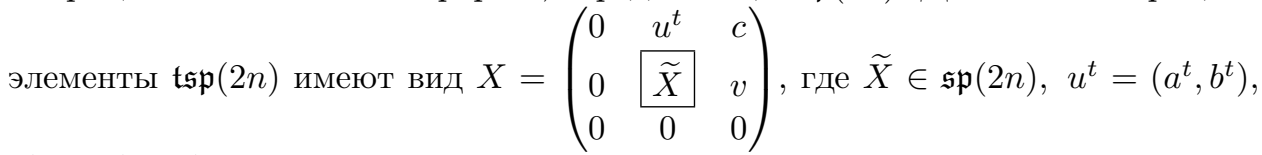
$v^{t}=\left(b^{t},-a^{t}\right), a, b \in \mathbb{C}^{n}$. Другими словами, $\mathfrak{t} \mathfrak{p}(2 n)$ является полупрямой суммой $\mathfrak{s p}(2 n)$ и алгебры Гейзенберга. Здесь и ниже мы опускаем нижние индексы $s, \gamma$, указывающие на точку $\gamma$, за исключением обозначения $z_{\gamma}$. В случаях 
$\mathfrak{g}=\mathfrak{s p}(2 n), \mathfrak{g}=\mathfrak{t} \mathfrak{p}(2 n)$ мы требуем, чтобы выполнялось равенство

$$
\alpha^{t} \sigma M_{1} \alpha=0
$$

Если указанные условия выполнены, то $M$ называется $\mathfrak{g}$-значным $M$-оператором. В [7] мы не накладывали на $M$-операторы условия (2.6), делая это только для $L$-операторов (см. раздел 3$)$.

Каждый $M$-оператор определяет касательный вектор к пространству данных Тюрина посредством дифференцирования координат:

$$
\partial_{M} z_{\gamma}=-\mu^{t} \sigma \alpha, \quad \partial_{M} \alpha=-M_{0} \alpha+\kappa \alpha,
$$

где $\kappa \in \mathbb{C}$ произвольно. Поскольку $\alpha$ задано с точностью до скалярного множителя, второе уравнение корректно определяет касательный вектор к соответствующему проективному пространству. Рассмотрим теперь $M$-операторы как функции $\{(\gamma, \alpha)\}$. Тогда соотношения $(2.7)$ определяют векторное поле на $T$.

Лемма 2.1. Для любых двух $M$-операторов $M_{a}, M_{b}$ и двух векторных полей $\partial_{a}, \partial_{b}$ на пространстве всех $M$-операторов, удовлетворяющих соотношениям (2.7), выражение

$$
M_{a b}=\partial_{a} M_{b}-\partial_{b} M_{a}+\left[M_{a}, M_{b}\right]
$$

также является $M$-оператором.

Для всех алгебр Ли из нашего списка за исключением $\mathfrak{g}=\mathfrak{s p}(2 n), \mathfrak{t s p}(2 n)$ лемма доказана в [7]. Мы воспроизводим здесь это доказательство, чтобы показать, в каком месте для $\mathfrak{g}=\mathfrak{t} \mathfrak{s}(2 n)$ требуются дополнительные соображения. Мы доказываем также, что $M_{a b}$ удовлетворяет соотношению (2.6), которое не использовалось в [7].

ДокАЗАТЕЛЬСтво. Проверим, что $M_{a b}$ Удовлетворяет условиям (2.4).

Для произвольной алгебры $\mathfrak{g}$ из нашего списка мы имеем следующее выражение для $M_{a}$ :

$$
M_{a}=\frac{\lambda_{a} \alpha \alpha^{t} \sigma}{\left(z-z_{\gamma}\right)^{2}}+\frac{\left(\alpha \mu_{a}^{t}+\varepsilon \mu_{a} \alpha^{t}\right) \sigma}{z-z_{\gamma}}+M_{0 a}+\cdots,
$$

и аналогичное выражение для $M_{b}$, где $\lambda_{a}, \lambda_{b}, \varepsilon$ и $\sigma$ определяются соотношениями (2.5) и последующими замечаниями. Далее,

$$
\begin{gathered}
\partial_{a} M_{b}=2\left(\partial_{a} z_{\gamma}\right) \frac{\lambda_{b} \alpha \alpha^{t} \sigma}{\left(z-z_{\gamma}\right)^{3}}+\frac{\left(\left(\partial_{a} \lambda_{b}\right) \alpha \alpha^{t}+\lambda_{b} \partial_{a}\left(\alpha \alpha^{t}\right)\right) \sigma+\left(\partial_{a} z_{\gamma}\right) M_{-1, b}}{\left(z-z_{\gamma}\right)^{2}} \\
+\frac{\left(\left(\partial_{a} \alpha\right) \mu_{b}^{t}+\varepsilon \mu_{b}\left(\partial_{a} \alpha^{t}\right)+\alpha\left(\partial_{a} \mu_{b}^{t}\right)+\varepsilon\left(\partial_{a} \mu_{b}\right) \alpha^{t}\right) \sigma}{z-z_{\gamma}}+\cdots,
\end{gathered}
$$

и аналогично для $\partial_{b} M_{a}$. 
Для коммутатора имеем

$$
\begin{aligned}
{\left[M_{a}, M_{b}\right]=} & \frac{\left(1+\varepsilon^{2}\right)\left(\lambda_{b} \cdot \mu_{a}^{t} \sigma \alpha-\lambda_{a} \cdot \mu_{b}^{t} \sigma \alpha\right) \alpha \alpha^{t} \sigma}{\left(z-z_{\gamma}\right)^{3}} \\
& +\frac{\left(\lambda_{a} \partial_{b}-\lambda_{b} \partial_{a}\right) \alpha \alpha^{t} \sigma+\lambda_{a b} \alpha \alpha^{t} \sigma+\left(\mu_{a}^{t} \sigma \alpha\right) M_{-1, b}-\left(\mu_{b}^{t} \sigma \alpha\right) M_{-1, a}}{\left(z-z_{\gamma}\right)^{2}} \\
& +\frac{\left(\left(\partial_{b} \alpha\right) \mu_{a}^{t}+\varepsilon \mu_{a}\left(\partial_{b} \alpha^{t}\right)\right) \sigma-\left(\left(\partial_{a} \alpha\right) \mu_{b}^{t}+\varepsilon \mu_{b}\left(\partial_{a} \alpha^{t}\right)\right) \sigma}{z-z_{\gamma}} \\
& +\frac{\left(\alpha \mu_{a b}^{t}+\varepsilon \mu_{a b} \alpha^{t}\right) \sigma}{z-z_{\gamma}}+\cdots,
\end{aligned}
$$

где

$$
\begin{aligned}
& \lambda_{a b}=2 \lambda_{b} \kappa_{a}-2 \lambda_{a} \kappa_{b}+\varepsilon\left(\mu_{a}^{t} \sigma \mu_{b}-\mu_{b}^{t} \sigma \mu_{a}\right), \\
& \mu_{a b}=\kappa_{a} \mu_{b}-\kappa_{b} \mu_{a}-\lambda_{a} M_{1 b} \alpha+\lambda_{b} M_{1 a} \alpha-M_{0 b} \mu_{a}+M_{0 a} \mu_{b} .
\end{aligned}
$$

Чтобы получить соотношение (2.9), мы воспользовались уравнениями (2.7) и некоторыми дополнительными соотношениями, в частности, соотношениями $\varepsilon \alpha^{t} \sigma \mu=-\varepsilon^{2} \mu^{t} \sigma \alpha$ и $\lambda \alpha^{t} \sigma \alpha=0$, которые выполнены во всех случаях. При вычислении $\left[M_{a}, M_{b}\right]_{-2}$ мы также пользовались соотношением

$$
\left[M_{-1, a}, M_{-1, b}\right]=\left(\mu_{a}^{t} \sigma \alpha\right) M_{-1, b}-\left(\mu_{b}^{t} \sigma \alpha\right) M_{-1, a}+\varepsilon\left(\mu_{a}^{t} \sigma \mu_{b}-\mu_{b}^{t} \sigma \mu_{a}\right) \alpha \alpha^{t} \sigma,
$$

которое может быть проверено с помощью (2.4). Чтобы получить $\left[M_{a}, M_{b}\right]_{-1}$ в форме $(2.9)$, существенно используется то, что $M_{i, a}^{t}=-\sigma M_{i, a} \sigma^{-1}$ для $\varepsilon \neq 0$ (что вытекает из (2.5)), и соответствующее равенство для $M_{i, b}$.

В случае $\mathfrak{g}=\mathfrak{t} \mathfrak{p}(2 n)$ необходимо доказать, что последняя координата вектора $\mu_{a b}$ равна нулю. Это очевидно так, поскольку матрицы $M_{1 a}, M_{1 b}, M_{0 a}, M_{0 b}$ имеют нулевые последние строки.

Сравнивая (2.9) и (2.8) (а также соответствующее соотношение для $\partial_{b} M_{a}$ ) и используя $(2.7)$, получаем

$$
M_{a b}=\frac{\widetilde{\lambda}_{a b} \alpha \alpha^{t} \sigma}{\left(z-z_{\gamma}\right)^{2}}+\frac{\left(\alpha \widetilde{\mu}_{a b}^{t}+\varepsilon \widetilde{\mu}_{a b} \alpha^{t}\right) \sigma}{z-z_{\gamma}}+\cdots,
$$

где $\widetilde{\lambda}_{a b}=\partial_{a} \lambda_{b}-\partial_{b} \lambda_{a}+\lambda_{a b}, \widetilde{\mu}_{a b}=\partial_{a} \mu_{b}-\partial_{b} \mu_{a}+\mu_{a b}$. Заметим, что $M_{a b}$ имеет вид $(2.3),(2.4)$. В частности, член порядка -3 исчезает, так как либо $\lambda_{a}=$ $\lambda_{b}=0$, либо $\varepsilon^{2}=1$ (что следует из (2.5)).

Докажем, что в симплектическом случае $M_{a b}$ удовлетворяет (2.6). Имеем

$$
\left(\partial_{a} M_{b}\right)_{1}=\partial_{a} M_{1 b}-2\left(\partial_{a} z_{\gamma}\right) M_{2 b}, \quad\left(\partial_{b} M_{a}\right)_{1}=\partial_{b} M_{1 a}-2\left(\partial_{b} z_{\gamma}\right) M_{2 a} .
$$

Применяя $\partial_{a}$ к обеим частям соотношения $\alpha^{t} \sigma M_{1 b} \alpha=0$ и $\partial_{b}$ к соответствующему соотношению для $M_{1 a}$, а также используя (2.7), находим

$$
\alpha^{t} \sigma\left(\partial_{a} M_{b 1}\right) \alpha=-\alpha^{t} \sigma\left[M_{0 a}, M_{1 b}\right] \alpha, \quad \alpha^{t} \sigma\left(\partial_{b} M_{a 1}\right) \alpha=-\alpha^{t} \sigma\left[M_{0 b}, M_{1 a}\right] \alpha .
$$

Далее, имеем

$$
\begin{aligned}
{\left[M_{a}, M_{b}\right]_{1}=\lambda_{a}[} & \left.\alpha \alpha^{t} \sigma, M_{3 b}\right]+\left[\left(\alpha \mu_{a}^{t}+\mu_{a} \alpha^{t}\right) \sigma, M_{2 b}\right]+\left[M_{0 a}, M_{1 b}\right] \\
& +\left[M_{1 a}, M_{0 b}\right]+\left[M_{a 2},\left(\alpha \mu_{b}^{t}+\mu_{b} \alpha^{t}\right) \sigma\right]+\lambda_{b}\left[M_{3 a}, \alpha \alpha^{t} \sigma\right]
\end{aligned}
$$


При вычислении $\alpha^{t} \sigma\left[M_{a}, M_{b}\right]_{1} \alpha$ первый и последний коммутаторы в правой части последнего соотношения дают нулевой вклад ввиду того, что $\alpha^{t} \sigma \alpha=0$ (точно так же, как и некоторые члены второго и пятого коммутаторов). Два коммутатора в середине сокращаются с коммутаторами в правых частях соотношений (2.11). Оставшиеся члены второго и пятого коммутаторов дают

$$
\begin{gathered}
\alpha^{t} \sigma\left(\mu_{a} \alpha^{t} \sigma M_{2 b}-M_{2 b} \alpha \mu_{a}^{t} \sigma+M_{2 a} \alpha \mu_{b}^{t} \sigma-\mu_{b} \alpha^{t} \sigma M_{2 a}\right) \alpha \\
=2 \alpha^{t} \sigma \mu_{a}\left(\alpha^{t} \sigma M_{2 b} \alpha\right)-2 \alpha^{t} \sigma \mu_{b}\left(\alpha^{t} \sigma M_{2 a} \alpha\right),
\end{gathered}
$$

что ввиду (2.7) сокращается с соответствующими членами выражения (2.10). Лемма доказана.

\section{3. $L$-операторы и алгебры операторов Лакса}

Мы определяем $L$-операторы как $M$-операторы, для которых уравнения (2.7) порождают тривиальную динамику. Таким образом, по определению, каждый $L$-оператор $L$ является мероморфной $\mathfrak{g}$-значной функцией на $\Sigma$, голоморфной вне $W \cup\left\{P_{+}, P_{-}\right\}$и такой, что в точках $\gamma=\gamma_{s}$

$$
L=\frac{L_{-2}}{\left(z-z_{\gamma}\right)^{2}}+\frac{L_{-1}}{z-z_{\gamma}}+L_{0}+\cdots,
$$

где $z$ - локальная координата в окрестности $\gamma, z_{\gamma}$ - координата самой точки $\gamma$, коэффициенты $L_{-2}, L_{-1}, L_{0}, L_{1}, \ldots$ принадлежат $\mathfrak{g}$ и

$$
L_{-2}=\nu \alpha \alpha^{t} \sigma, \quad L_{-1}=\left(\alpha \beta^{t}+\varepsilon \beta \alpha^{t}\right) \sigma,
$$

где $\nu \in \mathbb{C}, \beta \in \mathbb{C}^{n}, \sigma$ - матрица размера $n \times n$,

$$
\begin{array}{rlrlrl}
\nu \equiv 0, & \varepsilon=0, & \sigma=\mathrm{id} & & \text { для } \mathfrak{g}=\mathfrak{g l}(n), \mathfrak{s l}(n), \\
\nu \equiv 0, & \varepsilon=-1, & \sigma=\mathrm{id} & \text { для } \mathfrak{g}=\mathfrak{s o}(n), \\
& \varepsilon=1 & & \text { для } \mathfrak{g}=\mathfrak{s p}(2 n)
\end{array}
$$

и в последнем случае $\sigma$ - матрица симплектической формы. Случай $\mathfrak{g}=\mathfrak{t} \mathfrak{s}(2 n)$ в этом разделе не рассматривается, но такое рассмотрение возможно.

Условие тривиальности динамики (2.7) можно записать как

$$
\beta^{t} \sigma \alpha=0, \quad L_{0} \alpha=\kappa \alpha .
$$

Дополнительно мы предполагаем, что

$$
\begin{aligned}
\alpha^{t} \alpha=0 & \text { для } \mathfrak{g}=\mathfrak{s o}(n) \\
\alpha^{t} \sigma L_{1} \alpha=0 & \text { для } \mathfrak{g}=\mathfrak{s p}(2 n) .
\end{aligned}
$$

Теорема 3.1. Пространство $\overline{\mathfrak{g}}$ L-операторов является алгеброй Ли по отношению $к$ поточечному матричному коммутатору. Кроме того, для $\mathfrak{g}=$ $\mathfrak{g l}(n)$ оно является ассочиативной алгеброй относительно поточечного матричного умножения. 
Теорема доказана в [5]. Другое доказательство дано в [7], где теорема выводится из леммы 2.1. Ее несложно доказать и для $\mathfrak{g}=\mathfrak{t} \mathfrak{p} \mathfrak{p}(2 n)$. В последнем случае, используя уже доказанные для симплектического случая результаты, мы получаем, что если даны $L_{1}, L_{2} \in \overline{\mathfrak{s p}}(2 n+2)$, то коммутатор $\left[L_{1}, L_{2}\right]$ также принадлежит $\overline{\mathfrak{s p}}(2 n+2)$. Если $L_{1}, L_{2} \in \mathfrak{t} \mathfrak{p}(2 n)$ в каждой точке, то это верно и для $\left[L_{1}, L_{2}\right]$, и в силу доказательства леммы 2.1 соответствующий вектор $\beta$ имеет нулевую последнюю координату. Следовательно, $\left[L_{1}, L_{2}\right] \in \overline{\mathfrak{t s p}}(2 n)$.

Алгебра Ли $\overline{\mathfrak{g}}$ называется алгеброй операторов Лакса. Она зависит и от выбора параметров Тюрина, и от точек $P_{+}$и $P_{-}$, но мы опускаем в обозначениях всякое указание на эту зависимость.

Рассмотрим подробнее $\overline{\mathfrak{g l}}(n)$.

В этом случае $L_{-2}=0, L_{-1}=\alpha \beta^{t}$, где $\beta^{t} \alpha=0$ и $L_{0} \alpha=\kappa \alpha$. Эти ограничения означают, что элементы алгебры операторов Лакса $\overline{\mathfrak{g l}}(n)$ можно рассматривать как сечения расслоения эндоморфизмов $\operatorname{End}(B)$, где $B$ - голоморфное векторное расслоение, соответствующее данным Тюрина $T$.

Расщепление $\mathfrak{g l}(n)=\mathfrak{s}(n) \oplus \mathfrak{s l}(n)$, заданное отображением

$$
X \mapsto\left(\frac{\operatorname{tr}(X)}{n} I_{n}, X-\frac{\operatorname{tr}(X)}{n} I_{n}\right)
$$

$\left(\right.$ где $I_{n}-$ единичная матрица размера $\left.n \times n\right)$ индуцирует соответствующее расщепление для $\overline{\mathfrak{g l}}(n)$ :

$$
\overline{\mathfrak{g l}}(n)=\overline{\mathfrak{s}}(n) \oplus \overline{\mathfrak{s l}}(n) .
$$

Для $\overline{\mathfrak{s}}(n)$ все коэффициенты в $(3.1)$ - скалярные матрицы. Поэтому коэффициенты $L_{-1}$ равны нулю для всех $\gamma \in W$, т. е. элементы $\overline{\mathfrak{s}}(n)$ голоморфны на $W$. Матрица $L_{s, 0}$ скалярна и поэтому любое $\alpha_{s}$ - ее собственный вектор. Это означает, что по определению

$$
\overline{\mathfrak{s}}(n) \cong \mathfrak{s}(n) \otimes \mathscr{A} \cong \mathscr{A}
$$

(изоморфизм в смысле ассоциативных алгебр).

Любая алгебра операторов Лакса $\overline{\mathfrak{g}}$ допускает почти градуированную структуру (определение совмещено с теоремой 3.2 ниже).

Предположим, что все отмеченные точки (включая точки множества $W$ ) находятся в общем положении и $W \neq \varnothing$. Выберем локальные координаты $z_{ \pm}$ в окрестностях точек $P_{ \pm}$и $z_{s}$ в окрестностях точек $\gamma_{s}, s=1, \ldots, K$. Предположим, что $\mathfrak{g}-$ простая алгебра Ли из нашего списка. Для произвольного $m \in \mathbb{Z}$ рассмотрим подпространство

$$
\begin{aligned}
\overline{\mathfrak{g}}_{m}:= & \left\{L \in \overline{\mathfrak{g}} \mid \text { существуют } X_{+}, X_{-} \in \mathfrak{g}\right. \text { такие, что } \\
& \left.L\left(z_{+}\right)=X_{+} z_{+}^{m}+O\left(z_{+}^{m+1}\right), L\left(z_{-}\right)=X_{-} z_{-}^{-m-g}+O\left(z_{-}^{-m-g+1}\right)\right\} .
\end{aligned}
$$

Для $\mathfrak{g}=\mathfrak{g l}(n)$ выше доказано, что $\overline{\mathfrak{g l}}(n)=\overline{\mathfrak{s l}}(n) \oplus \mathscr{A} \cdot \mathrm{id}$, где $\mathscr{A}$ - алгебра функций Кричевера-Новикова. В этом случае мы полагаем

$$
\overline{\mathfrak{g l}}(n)_{m}=\overline{\mathfrak{s l}}(n)_{m} \oplus \mathscr{A}_{m} \cdot \mathrm{id},
$$


где $\mathscr{A}_{m}$ - соответствующее однородное подпространство для $\mathscr{A}$ [13]. Если $W=\varnothing$, мы попадаем в ситуацию алгебр Кричевера-Новикова и используем соответствующие аналоги условия (3.10) [13], [9].

Мы называем $\overline{\mathfrak{g}}_{m}$ (однороднъцм) подпространством степени $m$ в $\overline{\mathfrak{g}}$.

ТЕОРема 3.2 [5]. Подпространства $\overline{\mathfrak{g}}_{m}$ задают на $\overline{\mathfrak{g}}$ структуру почти градуированной алгебры Ли, а именно:

1) $\operatorname{dim} \overline{\mathfrak{g}}_{m}=\operatorname{dim} \mathfrak{g}$

2) $\overline{\mathfrak{g}}=\bigoplus_{m \in \mathbb{Z}} \overline{\mathfrak{g}}_{m}$;

3) $\left[\overline{\mathfrak{g}}_{m}, \overline{\mathfrak{g}}_{k}\right] \subseteq \bigoplus_{h=m+k}^{m+k+M} \overline{\mathfrak{g}}_{h}$, где $M=g$ для $\overline{\mathfrak{s l}}(n), \overline{\mathfrak{s o}}(n), \overline{\mathfrak{s p}}(2 n)$ u $M=g+1$ $\partial \bumpeq \Re \overline{\mathfrak{g l}}(n)$.

СлеДСтвиЕ 3.3. Пусть $X$ - элемент алгебры g. Для каждого $m$ существует единственный элемент $X_{m}$ алгебры $\overline{\mathfrak{g}}_{m}$ такой, что

$$
X_{m}=X z_{+}^{m}+O\left(z_{+}^{m+1}\right) .
$$

ДокАЗАТЕЛЬСтво. Из первого утверждения теоремы 3.2, т. е. из соотношения $\operatorname{dim} \overline{\mathfrak{g}}_{m}=\operatorname{dim} \mathfrak{g}$, следует, что существует единственная комбинация базисных элементов, для которой выполняется (3.12).

\section{4. g-значные уравнения Лакса}

В этом разделе мы рассматриваем вопросы согласованности и корректной определенности лаксовых уравнений вида

$$
L_{t}=[L, M], \quad L \in \overline{\mathfrak{g}}, \quad M \in \overline{\mathfrak{g}^{\diamond}},
$$

где $L, M$ - это $L$-оператор и $M$-оператор соответственно, а соответствие между $\mathfrak{g}$ и $\mathfrak{g}^{\diamond}$ задано следующим образом:

$$
\mathfrak{g}^{\diamond}= \begin{cases}\mathfrak{g l}(n), & \text { если } \mathfrak{g}=\mathfrak{g l}(n), \mathfrak{s l}(n), \\ \mathfrak{s o}(2 n+1), & \text { если } \mathfrak{g}=\mathfrak{s o}(2 n), \mathfrak{s o}(2 n+1), \\ \mathfrak{t} \mathfrak{p}(2 n), & \text { если } \mathfrak{g}=\mathfrak{s p}(2 n) .\end{cases}
$$

Во всех случаях предполагается, что $\mathfrak{g}$ вложена в $\mathfrak{g}^{\diamond}$ стандартным образом, таким образом, коммутатор $[L, M]$ определен.

Следуя [4], каждому эффективному дивизору $D=\sum_{i} m_{i} P_{i}$ на $\Sigma$ поставим в соответствие пространство $\mathscr{L}^{D}=\bigcup_{(\alpha, \gamma)} L_{\alpha, \gamma}^{D}$ (объединение по всем параметрам Тюрина $(\alpha, \gamma)$, удовлетворяющим условию $(3.5))$, где

$$
L_{\alpha, \gamma}^{D}=\left\{L \in \overline{\mathfrak{g}}_{\alpha, \gamma} \mid(L)+D \geqslant 0\right\}
$$

и $\overline{\mathfrak{g}}_{\alpha, \gamma}-$ алгебра операторов Лакса, соответствующая $(\alpha, \gamma)$. 
При выполнении условий, сформулированных ниже в лемме 4.2, уравнения Лакса (4.1) задают динамическую систему на $\mathscr{L}^{D}$.

Точкой сверху будем обозначать производную по времени, а термин " $\gamma$-точ$\kappa u "$ зарезервируем для точек $\gamma_{s}$.

ЛЕмма 4.1. В $\gamma$-точках из уравнений (4.1) вытекают следующие уравнения:

$$
\dot{z}_{\gamma}=-\mu^{t} \sigma \alpha, \quad \dot{\alpha}=-M_{0} \alpha+\kappa \alpha,
$$

совпадающие с уравнениями (2.7) с точностью до обозначений. Для $\mathfrak{g}=\mathfrak{s o}(n)$ утверждение верно лишь в общем положении (определенном в доказательстве).

ДокАЗАТЕЛЬСтво. Непосредственным вычислением получаем

$$
\begin{gathered}
\dot{L}=2 \dot{z}_{\gamma} \frac{\nu \alpha \alpha^{t} \sigma}{\left(z-z_{\gamma}\right)^{3}}+\frac{\dot{\nu} \alpha \alpha^{t} \sigma+\nu \dot{\alpha} \alpha^{t} \sigma+\nu \alpha \dot{\alpha}^{t} \sigma+\dot{z}_{\gamma}\left(\alpha \beta^{t}+\varepsilon \beta \alpha^{t}\right) \sigma}{\left(z-z_{\gamma}\right)^{2}} \\
+\frac{\dot{\alpha} \beta^{t} \sigma+\alpha \dot{\beta}^{t} \sigma+\varepsilon \dot{\beta} \alpha^{t} \sigma+\varepsilon \beta \dot{\alpha}^{t} \sigma}{z-z_{\gamma}}+\left(\dot{L}_{0}-\dot{z}_{\gamma} L_{1}\right)+\cdots
\end{gathered}
$$

Для $\mathfrak{g}=\mathfrak{g l}(n)$ мы должны взять $\nu=0, \varepsilon=0$. Решая уравнения (4.1) в классе рядов Лорана в точке $\gamma$, мы находим $\dot{z}_{\gamma}=-\mu^{t} \alpha$ в порядке -2 по $z-z_{\gamma}$, и

$$
\dot{\alpha} \beta^{t}+\alpha \dot{\beta}^{t}=\alpha \beta^{t} M_{0}-M_{0} \alpha \beta^{t}+\kappa \alpha \mu^{t}-\alpha \mu^{t} L_{0}
$$

в порядке -1 . Умножим обе части последнего соотношения на вектор $\widetilde{\beta}$, не ортогональный к $\beta$. Мы получим

$$
\dot{\alpha} \beta^{t} \widetilde{\beta}+\alpha \dot{\beta}^{t} \widetilde{\beta}=\alpha \beta^{t} M_{0} \widetilde{\beta}-M_{0} \alpha \beta^{t} \widetilde{\beta}+\kappa \alpha \mu^{t} \widetilde{\beta}-\alpha \mu^{t} L_{0} \widetilde{\beta} .
$$

Последнее можно разрешить относительно $\dot{\alpha}$, что дает

$$
\dot{\alpha}=-M_{0} \alpha+\widetilde{\kappa} \alpha, \quad \widetilde{\kappa} \in \mathbb{C} .
$$

В случае $\mathfrak{g}=\mathfrak{s o}(n)$ выбираем $\widetilde{\beta}$ ортогональным к $\alpha^{t}$ и $\dot{\alpha}^{t}-\alpha^{t} M_{0}$, и доказательство аналогично. Так как $\widetilde{\beta}$ не должно быть ортогонально к $\beta$, надо потребовать, чтобы $\beta$ не было равно никакой линейной комбинации $\alpha$ и $\dot{\alpha}-M_{0} \alpha$, что является условием общности положения.

В случае $\mathfrak{g}=\mathfrak{s p}(2 n)$ соотношение $\dot{z}_{\gamma}=-\mu^{t} \sigma \alpha$ вытекает из сравнения членов порядка -3 , а соотношение (4.5) следует из сравнения членов порядка -2 . В последнем случае мы получаем

$$
\begin{aligned}
& \alpha \alpha^{t} \sigma+\dot{\alpha} \alpha^{t} \sigma+\alpha \dot{\alpha}^{t} \sigma+\dot{z}_{\gamma}\left(\alpha \beta^{t}+\beta \alpha^{t}\right) \\
& \quad=\alpha \alpha^{t} \sigma M_{0}-M_{0} \alpha \alpha^{t} \sigma+\alpha \beta^{t} \sigma \mu \alpha^{t} \sigma-\alpha \mu^{t} \sigma \beta \alpha^{t} \sigma+\alpha\left(2 \kappa \alpha^{t} \sigma\right)+\dot{z}_{\gamma}\left(\alpha \beta^{t}+\beta \alpha^{t}\right) .
\end{aligned}
$$

Это соотношение легко разрешимо при помощи того же приема, что и выше (берется $\widetilde{\beta}$ такое, что $\alpha^{t} \sigma \beta \neq 0$ ). Лемма доказана.

Уравнения (4.3) - не что иное, как уравнения движения параметров Тюрина. Они существенно используются в [4]. Первоначально концепция деформации параметров Тюрина введена в [1], где она послужила для нахождения решений ранга выше 1 уравнений Кадомцева-Петвиашвили.

Обозначим через $T_{L} \mathscr{L}^{D}$ касательное пространство к $\mathscr{L}^{D}$ в точке $L$. 
Лемма 4.2. Следующие условия эквивалентнъ:

(i) $[L, M] \in T_{L} \mathscr{L}^{D}$,

(ii) $([L, M])+D \geqslant 0$ вне точек $\gamma$

при условии, что уравнения (4.3) выполнены в каждой точке $\gamma$.

ДокАзАТЕльство. В нашем доказательстве мы следуем работе [4], где лемма сформулирована и доказана для $\mathfrak{g}=\mathfrak{g l}(n)$.

Пусть $z$ - локальная координата в открытой окрестности, содержащей точку $\gamma$, а $z_{\gamma}-$ соответствующая координата $\gamma$.

Отождествим $T \mathscr{L}^{D}$ с пространством $\mathscr{T}^{D}$ всех мероморфных функций $T$ со значениями в $\mathfrak{g}$ таких, что в каждой точке $\gamma$

$$
\begin{gathered}
T=2 \dot{z}_{\gamma} \frac{\nu \alpha \alpha^{t} \sigma}{\left(z-z_{\gamma}\right)^{3}}+\frac{\dot{\nu} \alpha \alpha^{t} \sigma+\nu\left(\dot{\alpha} \alpha^{t}+\alpha \dot{\alpha}^{t}\right) \sigma+\dot{z}_{\gamma}\left(\alpha \beta^{t}+\varepsilon \beta \alpha^{t}\right) \sigma}{\left(z-z_{\gamma}\right)^{2}} \\
+\frac{\left(\dot{\alpha} \beta^{t}+\varepsilon \beta \dot{\alpha}^{t}+\alpha \dot{\beta}^{t}+\varepsilon \dot{\beta} \alpha^{t}\right) \sigma}{z-z_{\gamma}}+T_{0}+\cdots, \\
\dot{\alpha}^{t} \sigma \beta+\alpha^{t} \sigma \dot{\beta}=0, \\
T_{0} \alpha=\kappa \dot{\alpha}+\dot{\kappa} \alpha-L_{0} \dot{\alpha}-\dot{z}_{\gamma} L_{1} \alpha,
\end{gathered}
$$

где $\dot{z}_{\gamma}, \dot{\kappa}$ - константы, $\dot{\alpha}, \dot{\beta}$ - постоянные векторы, удовлетворяющие соотношениям (4.3), и дивизор $T$ вне точек $\gamma$ больше или равен дивизору $-D$.

Соотношение (4.6) моделирует соотношение (4.4), полученное путем дифференцирования по времени равенства (3.1). В частности,

$$
T_{0}=\dot{L}_{0}-\dot{z}_{\gamma} L_{1}
$$

Вместе с дифференцированием по времени второго из соотношений (3.4) это дает (4.8). Таким образом, $T \mathscr{L}^{D}$ вкладывается в $\mathscr{T}^{D}$. Проверим совпадение размерностей этих пространств. Это делается более или менее одинаково для всех типов алгебр, так что мы проделаем это в наиболее трудном случае $\mathfrak{g}=$ $\mathfrak{s p}(2 n)$. Имеем

$$
(T)+\widetilde{D} \geqslant 0, \text { где } \widetilde{D}=D+3 \sum \gamma, \quad \text { и } \quad \operatorname{deg} \widetilde{D}=\operatorname{deg} D+3 K .
$$

По теореме Римана-Роха $\operatorname{dim}\{T \mid(T)+\widetilde{D} \geqslant 0\}=(\operatorname{dim} \mathfrak{g})(\operatorname{deg} D+3 K-g+1)$. Элементы пространства $\mathscr{T}^{D}$ выделяются в пространстве $\{T \mid(T)+\widetilde{D} \geqslant 0\}$ следующими соотношениями. Во-первых, в каждой точке $\gamma$ мы имеем

$$
\begin{aligned}
& T_{-3}=2 \dot{z}_{\gamma} \nu \alpha \alpha^{t} \sigma \\
& T_{-2}=\dot{\nu} \alpha \alpha^{t} \sigma+\nu\left(\dot{\alpha} \alpha^{t}+\alpha \dot{\alpha}^{t}\right) \sigma+\dot{z}_{\gamma}\left(\alpha \beta^{t}+\beta \alpha^{t}\right) \sigma, \\
& T_{-1}=\left(\dot{\alpha} \beta^{t}+\alpha \dot{\beta}^{t}+\dot{\beta} \alpha^{t}+\beta \dot{\alpha}^{t}\right) \sigma .
\end{aligned}
$$

Так как элементы в левой части равенства принадлежат $\mathfrak{g}$, то (4.9) дает $3 \operatorname{dim} \mathfrak{g}$ соотношений. Равенства (4.7) и (4.8) дают $2 n+1$ соотношений, и еще $2 n+1$ 
соотношений получаем из (4.3). Таким образом, мы имеем $3 \operatorname{dim} \mathfrak{g}+4 n+2$ соотношений. Они содержат $4 n+2$ свободных параметров $\dot{z}_{\gamma}, \dot{\nu}, \dot{\alpha}, \dot{\beta}$. Таким образом, мы фактически получили $3 \operatorname{dim} \mathfrak{g}$ соотношений в каждой точке $\gamma$, а число этих точек равно $K$, следовательно, мы имеем $3(\operatorname{dim} \mathfrak{g}) K$ соотношений. Мы видим, что

$$
\operatorname{dim} \mathscr{T}^{D}=(\operatorname{dim} \mathfrak{g})(\operatorname{deg} D-g+1)
$$

Но $\mathscr{L}^{D}$ имеет такую же размерность. Это можно вычислить аналогично или воспользоваться теоремой 3.2. Предположим, что мы находимся в двухточечной ситуации, т. е. $D=-m_{+} P_{+}+\left(m_{-}+g\right) P_{-}$, где для простоты $m_{-}>m_{+}$. Тогда $\mathscr{L}^{D}=\mathfrak{g}_{m_{+}} \oplus \cdots \oplus \mathfrak{g}_{m_{-}}$. По теореме $3.2 \operatorname{dim} \mathscr{L}^{D}=(\operatorname{dim} \mathfrak{g})\left(m_{-}-m_{+}+1\right)$, что в точности равно $(\operatorname{dim} \mathfrak{g})(\operatorname{deg} D-g+1)$. Мы заключаем, что $\operatorname{dim} \mathscr{T}^{D}=$ $\operatorname{dim} T_{L} \mathscr{L}^{D}$, следовательно, эти линейные пространства совпадают.

Далее мы доказываем, что если $L, M$ - те же, что и выше, то $[L, M]$ обладает свойствами (4.6)-(4.8), т. е. принадлежит пространству $\mathscr{T}^{D}$. Доказательство снова непосредственное. Например, проверим (4.8). Обозначим член нулевой степени $[L, M]_{0}$ коммутатора через $T_{0}$. Тогда в случае $\mathfrak{g}=\mathfrak{g l}(n)$ находим с помощью вычислений, что

$$
T_{0} \alpha=\alpha\left(\beta^{t} M_{1} \alpha-\mu^{t} L_{1} \alpha\right)+\left(L_{0}-\kappa\right) M_{0} \alpha+L_{1} \alpha\left(\mu^{t} \alpha\right) .
$$

Если заменить $\mu^{t} \alpha$ на $-\dot{z}_{\gamma}, M_{0} \alpha$ на $-\dot{\alpha}$ и обозначить $\beta^{t} M_{1}-\mu^{t} L_{1} \alpha$ через $\dot{\kappa}$, получим (4.8). Для других типов алгебр $\mathfrak{g}$ выражение для $T_{0} \alpha$ сложнее, и чтобы отождествить его с (4.8), мы пользуемся соотношениями (3.4)-(3.6).

В случае $\mathfrak{g}=\mathfrak{s p}(2 n)$ вместо (4.10) мы получаем

$$
\begin{aligned}
T_{0} \alpha=\alpha & \left(\nu \alpha^{t} \sigma M_{2} \alpha+\beta^{t} \sigma M_{1} \alpha-\mu^{t} \sigma L_{1} \alpha-\lambda \alpha^{t} \sigma L_{2} \alpha\right) \\
& +\left(L_{0}-\kappa\right) M_{0} \alpha+L_{1} \alpha\left(\mu^{t} \sigma \alpha\right)+\beta \alpha^{t} \sigma M_{1} \alpha-\mu \alpha^{t} \sigma L_{1} \alpha .
\end{aligned}
$$

Пользуясь соотношениями $\alpha^{t} \sigma M_{1} \alpha=0$ и $\alpha^{t} \sigma L_{1} \alpha=0$, мы получаем тот же результат. Лемма доказана.

Из леммы 4.2 непосредственно вытекает, что если $([L, M])+D \geqslant 0$ вне точек $\gamma$ и уравнения движения полюсов выполнены, то уравнения Лакса (4.1) имеют смысл.

\section{5. Иерархии коммутирующих потоков}

В случае $\mathfrak{g}=\mathfrak{g l}(n)$ И. М. Кричевер [4] показал, что оператор Лакса, рассматриваемый как функция на $\mathscr{L}^{D}$, порождает на $\mathscr{L}^{D}$ иерархию коммутирующих потоков. Этот результат допускает различные обобщения на классические алгебры Ли.

В этой статье мы рассмотрим два метода построения иерархий коммутирующих потоков по оператору Лакса. Они отличаются пространствами $M$-операторов и числом $\gamma$-точек. С помощью первого метода мы получим ниже (следуя [4]) эллиптическую систему Калоджеро-Мозера $A_{n}$. Вторым методом мы получим эллиптические системы Калоджеро-Мозера $C_{n}$ и $D_{n}$. 
Первый метод таков. Определим для дивизора $D=\sum m_{i} P_{i}$ дивизор $\widetilde{D}=$ $D+\delta \sum_{s=1}^{K} \gamma_{s}$, где

$$
K= \begin{cases}n g, & \mathfrak{g}=\mathfrak{g l}(n), \mathfrak{s l}(n), \mathfrak{s o}(2 n), \mathfrak{s o}(2 n+1), \\ (n+1) g, & \mathfrak{g}=\mathfrak{s p}(2 n),\end{cases}
$$

и

$$
\delta= \begin{cases}1, & \mathfrak{g}=\mathfrak{g l}(n), \mathfrak{s l}(n), \mathfrak{s o}(2 n), \mathfrak{s o}(2 n+1), \\ 2, & \mathfrak{g}=\mathfrak{s p}(2 n)\end{cases}
$$

$\left(K\right.$ и $\delta$ в действительности зависят от $\left.\mathfrak{g}^{\diamond}\right)$. $\widetilde{D} \geqslant 0$.

Определим $\mathscr{N}^{D} \subset \overline{\mathfrak{g}^{\diamond}}$ как подпространство $M$-операторов таких, что $(M)+$

Лемма 5.1. $\operatorname{dim} \mathscr{N}^{D}=\left(\operatorname{dim} \mathfrak{g}^{\diamond}\right)(\operatorname{deg} D+1)$.

ДоказАтельство. Мы вычисляем $\operatorname{dim} \mathscr{N}^{D}$ с помощью теоремы РиманаРоха с учетом дополнительных соотношений в точках $\gamma$. Это соотношения, определяющие $M_{-2}, M_{-1}$ и $M_{1}$ (последнее только в случае $\left.\mathfrak{g}=\mathfrak{s p}(2 n)\right)$. Число этих соотношений в каждой точке $\gamma$ равно $\delta \operatorname{dim} \mathfrak{g}^{\diamond}$, если $\mathfrak{g} \neq \mathfrak{s} \mathfrak{p}(2 n)$, и $\delta \operatorname{dim} \mathfrak{g}^{\diamond}+1$ в противоположном случае. Мы записываем это число в виде $\delta \operatorname{dim} \mathfrak{g}^{\diamond}+r_{\mathfrak{s p}}$, где $r_{\mathfrak{s p}}=1$ для $\mathfrak{g}=\mathfrak{s p}(2 n)$ и $r_{\mathfrak{s p}}=0$ в остальных случаях. У нас имеются свободные параметры $\mu, \lambda(\lambda$ возникает только в случае $\mathfrak{g}=\mathfrak{s p}(2 n))$. Пусть $r-$ число этих параметров при фиксированном $\gamma$. Мы можем считать, что имеется $\delta \operatorname{dim} \mathfrak{g}-r+r_{\mathfrak{s p}}$ соотношений для каждого $\gamma$.

Запишем $K$ в виде $K=l g$, где $l$ равно $n$ или $n+1$ в зависимости от $\mathfrak{g}^{\diamond}$. Имеем

$$
\begin{aligned}
\operatorname{dim} \mathscr{N}^{D} & =\left(\operatorname{dim} \mathfrak{g}^{\diamond}\right)(\operatorname{deg} D+\delta l g-g+1)-\left(\delta \operatorname{dim} \mathfrak{g}^{\diamond}-r+r_{\mathfrak{s p}}\right) l g \\
& =\left(\operatorname{dim} \mathfrak{g}^{\diamond}\right)(\operatorname{deg} D+1)-\left(\operatorname{dim} \mathfrak{g}^{\diamond}-\left(r-r_{\mathfrak{s p}}\right) l\right) g .
\end{aligned}
$$

Теперь проверим, что

$$
\operatorname{dim} \mathfrak{g}^{\diamond}=\left(r-r_{\mathfrak{s p}}\right) l .
$$

В самом деле, для $\mathfrak{g}^{\diamond}=\mathfrak{g l}(n)$ мы имеем $r=l=n, r_{\mathfrak{s p}}=0$, следовательно, $\left(r-r_{\mathfrak{s p}}\right) l=n^{2}$. Если $\mathfrak{g}^{\diamond}=\mathfrak{s o}(2 n+1)$, то $r=2 n+1, l=n, r_{\mathfrak{s p}}=0$ и $\left(r-r_{\mathfrak{s p}}\right) l=$ $(2 n+1) n$. Наконец, если $\mathfrak{g}^{\diamond}=\mathfrak{t} \mathfrak{s} \mathfrak{p}(2 n)$, то $\operatorname{dim} \mathfrak{g}^{\diamond}=n(2 n+1)+(2 n+1)=(2 n+1) \times$ $(n+1)$ (сумма размерностей $\mathfrak{s p}(2 n)$ и алгебры Гейзенберга, см. с. 155). В этом случае $r=2 n+2(2 n+1$ свободных параметров дает $\mu$ и один соответствует $\lambda)$. Следовательно, $r-r_{\mathfrak{s p}}=2 n+1$ и $\left(r-r_{\mathfrak{s p}}\right) l=(2 n+1)(n+1)$.

Во всех случаях соотношение (5.2) выполнено. Лемма доказана.

Следуя [4], зафиксируем точку $P_{0} \in \Sigma$ и локальные координаты $w_{0}, w_{i}$ в окрестностях точек $P_{0}, P_{i}$. Наша следующая цель - определить калибровочно инвариантные функции $M_{a}$, удовлетворяющие предположениям леммы 4.2. Определим $a$ как тройку

$$
a=\left(P_{i}, k, m\right), \quad k>0, \quad m>-m_{i},
$$

где $k, m$ - целые, $k \equiv 1(\bmod 2)$ для $\mathfrak{g}=\mathfrak{s o}(n)$ и $\mathfrak{g}=\mathfrak{s p}(2 n)$. 
По лемме 5.1 в общем положении для $L \in \overline{\mathfrak{g}}$ имеется единственная $\mathfrak{g}^{\diamond}$-значная мероморфная функция $M_{a}$ такая, что

(i) $M_{a}$ является $M$-оператором;

(ii) вне точек $\gamma$ она имеет полюс только в точке $P_{i}$ и

$$
M_{a}(q)=w_{i}^{-m} L^{n}(q)+O(1),
$$

т. е. главные части $M_{a}$ и $w_{i}^{-m} L^{n}$ совпадают;

(iii) $M_{a}$ нормализована условием $M_{a}\left(P_{0}\right)=0$.

Теорема 5.2. Уравнения

$$
\partial_{a} L=\left[L, M_{a}\right], \quad \partial_{a}=\frac{\partial}{\partial t_{a}},
$$

определяют иерархию коммутирующих потоков на открытом множестве в $\mathscr{L}^{D}$.

Для $\mathfrak{g}=\mathfrak{g l}(n)$ теорема сформулирована и доказана в [4]. Для $\mathfrak{g}=\mathfrak{s o}(2 n)$, $\mathfrak{s o}(2 n+1)$ она доказана в [7] при несколько иных предположениях. Здесь мы формулируем и доказываем теорему для всех классических алгебр Ли, включая $\mathfrak{g}=\mathfrak{s p}(2 n)$.

ДокАЗАТЕЛЬство. Из (ii) следует, что $\left(\left[L, M_{a}\right]\right)+D \geqslant 0$, следовательно, по лемме $4.2\left[L, M_{a}\right] \in T_{L} \mathscr{L}^{D}$ и уравнение $\partial_{a} L=\left[L, M_{a}\right]$ определяет поток на $\mathscr{L}^{D}$.

Для доказательства коммутативности таких потоков достаточно проверить, что $M_{a b}=\partial_{a} M_{b}-\partial_{b} M_{a}+\left[M_{a}, M_{b}\right]=0$ тождественно. Прежде всего, по лемме 4.1 векторные поля $\partial_{a}, \partial_{b}$ удовлетворяют соотношениям (2.7). Следовательно, по лемме $2.1 M_{a b}$ является $M$-оператором. Ниже мы показываем, что этот $M$-оператор регулярен в точках дивизора $D$. По лемме 5.1 пространство таких операторов имеет ту же размерность, что и $\mathfrak{g}^{\diamond}$. Ввиду (iіi) получаем $M_{a b}=0$.

Докажем, что $M_{a b}$ регулярен в точках дивизора $D$. Мы повторяем здесь соответствующую часть доказательства теоремы 2.1 работы [4]. Сначала предположим, что индексы $a, b$ соответствуют одной и той же точке $P_{i}$, т. е. $a=$ $\left(P_{i}, n, m\right), b=\left(P_{i}, n^{\prime}, m^{\prime}\right)$. Обозначим $M_{a}-w^{-m} L^{n}$ через $M_{a}^{-}$и $M_{b}-w^{-m^{\prime}} L^{n^{\prime}}$ через $M_{b}^{-}$, тогда ввиду (ii) $M_{a}^{-}$и $M_{b}^{-}$регулярны в окрестности $P_{i}$. Имеем

$$
\begin{aligned}
\partial_{a} M_{b} & =w^{-m^{\prime}} \partial_{a} L^{n^{\prime}}+\partial_{a} M_{b}^{-}=w^{-m^{\prime}}\left[L^{n^{\prime}}, M_{a}\right]+\partial_{a} M_{b}^{-} \\
& =w^{-m^{\prime}}\left[L^{n^{\prime}}, M_{a}^{-}\right]+\partial_{a} M_{b}^{-}
\end{aligned}
$$

И

$$
\begin{aligned}
{\left[M_{a}, M_{b}\right] } & =\left[M_{a}^{-}+w^{-m} L^{n}, M_{b}^{-}+w^{-m^{\prime}} L^{n^{\prime}}\right] \\
& =w^{-m}\left[L^{n}, M_{b}^{-}\right]-w^{-m^{\prime}}\left[L^{n^{\prime}}, M_{a}^{-}\right]+\left[M_{a}^{-}, M_{b}^{-}\right] .
\end{aligned}
$$

Следовательно,

$$
M_{a b}=\partial_{a} M_{b}^{-}-\partial_{b} M_{a}^{-}+\left[M_{a}^{-}, M_{b}^{-}\right],
$$

что является выражением, регулярным в точке $P_{i}$. По определению $M_{a b}$ регулярно также в других точках дивизора $D$.

В случае, когда $a$ и $b$ соответствуют разным точкам дивизора $D$, доказательство аналогично. Теорема доказана. 


\section{6. Симплектическая структура}

В этом разделе, следуя [4], мы вводим симплектическую структуру на некотором подпространстве $\mathscr{P}^{D} \subset \mathscr{L}^{D} / G$, где $G=\exp \mathfrak{g}$. Мы называем ее симплектической структурой Кричевера-Фонга.

Пуст $\Psi$ - матрица, составленная из канонически нормализованных левых собственных векторов $L$ (под канонической нормализацией вектора $\psi$ мы понимаем соотношение $\left.\sum \psi_{i}=1\right)$. Она определена с точностью до перестановки строк. Мы рассматриваем $L$ и $\Psi$ как матричнозначные функции на $\mathscr{L}^{D}$. Пусть $\delta L$ и $\delta \Psi$ - их внешние дифференциалы, которые являются 1-формами на $\mathscr{L}^{D}$. Аналогично мы рассматриваем диагональную матрицу $K$, определенную соотношением

$$
\Psi L=K \Psi
$$

т. е. образованную собственными значениями $L$, и матричнозначную 1 -форму $\delta K$. Пусть $\Omega-2$-форма на $\mathscr{L}^{D}$, принимающая значения в пространстве мероморфных функций на $\Sigma$, определенная соотношением

$$
\Omega=\operatorname{tr}\left(\delta \Psi \wedge \delta L \cdot \Psi^{-1}-\delta K \wedge \delta \Psi \cdot \Psi^{-1}\right)
$$

Форма $\Omega$ не зависит от от порядка собственных значений и, таким образом, корректно определена на $\mathscr{L}$.

Зафиксируем голоморфный дифференциал $d z$ на $\Sigma$ и определим скалярную 2-форму $\omega$ на $\mathscr{L}^{D}$ при помощи соотношения

$$
\omega=-\frac{1}{2}\left(\sum_{s=1}^{K} \operatorname{res}_{\gamma_{s}} \Omega d z+\sum_{P_{i} \in D} \Omega d z\right) .
$$

Форма $\Omega$ имеет и другое представление:

$$
\Omega=2 \delta \operatorname{tr}\left(\delta \Psi \cdot \Psi^{-1} K\right),
$$

из которого очевидным образом следует, что $\omega$ замкнута.

Перейдем к исследованию невырожденности формы $\omega$. Сначала докажем, что она невырождена при ограничении на пространство параметров Тюрина, т. е. определяет симплектическую форму на этом пространстве. Мы укажем каноническую форму этого ограничения.

Лемма 6.1. Вклад параметров Тюрина в $ш$ имеет вид

$$
\omega_{0}=\sum_{s=1}^{K}\left(a \delta z_{s} \wedge \delta \kappa_{s}+\delta \beta_{s}^{t} \wedge \delta \alpha_{s}\right),
$$

где $a=1$ для $\mathfrak{g}=\mathfrak{g l}(n), a=2$ для $\mathfrak{g}=\mathfrak{s o}(n) u \mathfrak{g}=\mathfrak{s p}(2 n)$.

ДокАЗАТЕЛЬСтво. Для $\mathfrak{g}=\mathfrak{g l}(n)$ соответствующее утверждение содержится в леммах 2.1 и 4.3 работы [4]. Полезно воспроизвести его доказательство здесь. Пусть $g_{s}-$ постоянная по $z$ невырожденная матрица такая, что $g_{s} \alpha=e_{1}$, где $e_{1}^{t}=(1,0, \ldots, 0)$. Тогда для $L_{s}^{\prime}=g_{s} L_{s} g_{s}^{-1}$ имеем $\left(L_{s}^{\prime}\right)_{-1}=e_{1} f^{t}$, где 
$f^{t}=\beta^{t} g_{s}^{-1}$, и $f_{1}=0$, так как $f^{t} e_{1}=0$. Очевидно, что только элементы первой строки матрицы $L_{s,-1}^{\prime}$ могут быть ненулевыми, при этом $\left(L_{s,-1}^{\prime}\right)_{11}=0$.

Вектор $e_{1}$ является собственным для $L_{s 0}^{\prime}$ с собственным значением $\kappa$. По этой причине $\left(L_{s 0}^{\prime}\right)^{11}=\kappa,\left(L_{s 0}^{\prime}\right)^{i 1}=0, i>1$.

Таким образом, сопряжение матрицей $f_{s}=\operatorname{diag}\left(z-z_{s}, 1, \ldots, 1\right)$ переводит $\left(z-z_{s}\right)^{-1} L_{s,-1}^{\prime}$ и $L_{s 0}^{\prime}$ в голоморфные матричнозначные функции. Следовательно, матричная функция $f_{s} L_{s}^{\prime} f_{s}^{-1}$ голоморфна. Заметим на будущее, что $f_{s}$ диагональна.

Проследим теперь за преобразованиями формы $\Omega$, соответствующими только что выполненным преобразованиям $L$.

Под действием калибровочного преобразования $L^{\prime}=g L g^{-1}, \Psi^{\prime}=\Psi g^{-1}$ форма $\Omega$ преобразуется к виду $\Omega^{\prime}$, где $\Omega^{\prime}=\Omega+2 \operatorname{tr}\left(\delta L \wedge g^{-1} \delta g-L g^{-1} \delta g \wedge g^{-1} \delta g\right)$.

При $g=g_{s}$ мы получим

$$
\operatorname{res}_{\gamma_{s}} \Omega^{\prime} d z=\operatorname{res}_{\gamma_{s}} \Omega d z+2 \operatorname{res}_{\gamma_{s}} \operatorname{tr}\left(\delta L \wedge g_{s}^{-1} \delta g_{s}-L g_{s}^{-1} \delta g_{s} \wedge g_{s}^{-1} \delta g_{s}\right) d z .
$$

Так как $g_{s}$ постоянна по $z$, имеем

$\operatorname{res}_{\gamma_{s}} \Omega^{\prime} d z=\operatorname{res}_{\gamma_{s}} \Omega d z+2 \operatorname{tr}\left(\left(\delta \alpha_{s} \cdot \beta_{s}^{t}+\alpha_{s} \cdot \delta \beta_{s}^{t}\right) \wedge g_{s}^{-1} \delta g_{s}-\alpha_{s} \beta_{s}^{t} g_{s}^{-1} \delta g_{s} \wedge g_{s}^{-1} \delta g_{s}\right)$.

Дифференцируя соотношение $g_{s} \alpha_{s}=e_{1}$, находим, что $\delta \alpha_{s}=-g_{s}^{-1} \delta g_{s} \alpha_{s}$. Подставляя это в предыдущее соотношение, получаем

$$
\operatorname{res}_{\gamma_{s}} \Omega^{\prime} d z=\operatorname{res}_{\gamma_{s}} \Omega d z+2 \operatorname{tr}\left(\delta \alpha_{s} \wedge \delta \beta_{s}^{t}\right) .
$$

Матрица $L_{s}^{\prime}$ в результате преобразования $\widehat{L}_{s}=f_{s} L_{s}^{\prime} f_{s}^{-1}$ становится голоморфной, следовательно,

$$
0=\operatorname{res}_{\gamma_{s}} \Omega^{\prime} d z+2 \operatorname{res}_{\gamma_{s}} \operatorname{tr}\left(\delta L^{\prime} \wedge f_{s}^{-1} \delta f_{s}-L^{\prime} \delta f_{s}^{-1} f_{s} \wedge \delta f_{s}^{-1} f_{s}\right) .
$$

Так как $f_{s}$ диагональна, то последний член обращается в нуль. Воспользовавшись найденным выше специальным видом $L_{s}^{\prime}$ и $f_{s}$, заключаем, что

$$
\operatorname{res}_{\gamma_{s}} \Omega^{\prime} d z=-2 \delta z_{s} \wedge \delta \kappa_{s}
$$

Таким образом, вклад точки $\gamma_{s}$ в $\Omega$ равен

$$
\operatorname{res}_{\gamma_{s}} \Omega=-2 \operatorname{tr}\left(\delta \alpha_{s} \wedge \delta \beta_{s}^{t}\right)-2 \delta z_{s} \wedge \delta \kappa_{s},
$$

а соответствующий вклад в $\omega$ равен

$$
\omega_{s}=-\frac{1}{2} \operatorname{res}_{\gamma_{s}} \Omega=\delta \beta_{s}^{t} \wedge \delta \alpha_{s}+\delta z_{s} \wedge \delta \kappa_{s}
$$

Доказательство для $\mathfrak{s o}(n)$ и $\mathfrak{s p}(2 n)$ аналогично, но сложнее. В симплектическом случае мы выбираем матрицу, задающую симплектическую форму, в виде

$$
\sigma=\left(\begin{array}{cc}
\sigma^{\prime} & 0 \\
0 & \sigma^{\prime \prime}
\end{array}\right), \quad \text { где } \sigma^{\prime}=\left(\begin{array}{cc}
0 & 1 \\
-1 & 0
\end{array}\right) .
$$


Мы можем перевести вектор $\alpha$ в любой другой вектор симплектическим преобразованием $g$. Переведем его в $e_{1}$, где $e_{1}^{t}=(1,0, \ldots, 0)^{t}$. Тогда

$$
\alpha \alpha^{t} \sigma=\left(\begin{array}{ccccc}
0 & 1 & 0 & \ldots & 0 \\
0 & 0 & 0 & \ldots & 0 \\
0 & 0 & 0 & \ldots & 0 \\
\vdots & \vdots & \vdots & \ddots & \vdots \\
0 & 0 & 0 & \ldots & 0
\end{array}\right), \quad\left(\alpha \beta^{t}+\beta \alpha^{t}\right) \sigma=\left(\begin{array}{ccccc}
-\beta_{2} & 2 \beta_{1} & * & \ldots & * \\
0 & \beta_{2} & 0 & \ldots & 0 \\
0 & \beta_{3} & 0 & \ldots & 0 \\
\vdots & \vdots & \vdots & \ddots & \vdots \\
0 & \beta_{2 n} & 0 & \ldots & 0
\end{array}\right),
$$

где звездочки обозначают константы, линейно зависящие от $\beta$. Заметим, что ввиду $\beta^{t} \sigma \alpha=0$ имеем $\beta_{2}=0$.

Несложно проверить, что сопряжение матрицей $f=\left(\begin{array}{cc}Z & 0 \\ 0 & E\end{array}\right), \quad$ где $Z=\left(\begin{array}{cc}z & 0 \\ 0 & z^{-1}\end{array}\right)$, умножает единственный ненулевой элемент матрицы $\alpha \alpha^{t} \sigma$ на $z^{2}$, а матрицу $\left(\alpha \beta^{t}+\beta \alpha^{t}\right) \sigma-$ на $z$ (за исключением элемента $2 \beta_{1}$, который умножается на $\left.z^{2}\right)$. Следовательно, $f\left(\frac{\alpha \alpha^{t} \sigma}{z^{2}}+\frac{\left(\alpha \beta^{t}+\beta \alpha^{t}\right) \sigma}{z}\right) f^{-1}-$

Матрица $L_{0}$ по структуре аналогична $\left(\alpha \beta^{t}+\beta \alpha^{t}\right) \sigma$. Ее первый столбец равен $(\kappa, 0, \ldots, 0)^{t}$, так как $L_{0} e_{1}=\kappa e_{1}$. Ее вторая строка равна $(0,-\kappa, 0, \ldots, 0)^{t}$, так как $\left(e_{1}^{t} \sigma\right) L_{0}=-\kappa\left(e_{1}^{t} \sigma\right)$ (что в свою очередь следует из соотношения $L_{0}^{t} \sigma+$ $\left.\sigma L_{0}=0\right)$. При сопряжении матрицей $f$ ненулевые элементы $L_{0}$ умножаются на положительные степени $z$, за исключением правого нижнего углового блока размера $(2 n-2) \times(2 n-2)$, который остается инвариантным. Следовательно, матричная функция $f L_{0} f^{-1}$ голоморфна.

Особенность могла бы возникнуть из $f\left(z L_{1}\right) f^{-1}$, поскольку $\left(L_{1}\right)_{21}$ умножается на $z^{-2}$, но из $\alpha^{t} \sigma L_{1} \alpha=0$ следует $\left(L_{1}\right)_{21}=0$. Все остальные элементы матрицы $f L_{1} f^{-1}$ имеют порядок $z^{-1}$ и выше и, следовательно, после умножения на $z$ становятся голоморфными.

Все элементы матрицы $\delta f \cdot f^{-1}$ равны нулю за исключением левого верхнего углового блока размера $2 \times 2$, который равен $\left(\begin{array}{cc}\delta z \cdot z^{-1} & 0 \\ 0 & -\delta z \cdot z^{-1}\end{array}\right)$. Как вытекает из сказанного о структуре матрицы $L_{0}$, соответствующий блок матрицы $\delta L_{0}$ равен $\left(\begin{array}{cc}\delta \kappa & * \\ 0 & -\delta \kappa\end{array}\right)$. Таким образом, в точке $\gamma_{s}$ мы имеем вклад $\delta \beta_{s}^{t} \wedge \delta \alpha_{s}$ от калибровочного преобразования, соответствующего $g$, и вклад $2 \delta z_{s} \wedge \delta \gamma_{s}$ от калибровочного преобразования, соответствующего $f$. Общий вклад точки $\gamma_{s}$ в $\omega$ равен $2 \delta z_{s} \wedge \delta \kappa_{s}+\delta \beta_{s}^{t} \wedge \delta \alpha_{s}$.

В ортогональном случае мы должны дополнительно обеспечить выполнение условия $e_{1}^{t} \sigma e_{1}=0$. Рассмотрим, например, $\mathfrak{g}=\mathfrak{s o}(2 n)$. Выберем $\sigma$ аналогично симплектическому случаю, но с $\sigma^{\prime}=\left(\begin{array}{ll}0 & 1 \\ 1 & 0\end{array}\right)$ и положительно определенной матрицей $\sigma^{\prime \prime}$. Тогда $e_{1}$ (где $\left.e_{1}^{t}=(1,0, \ldots, 0)^{t}\right)$ удовлетворяет требуемому соотношению и мы действуем как в симплектическом случае, с той же самой матричной функцией $f$. Структура $L_{-1}=\left(\alpha \beta^{t}-\beta \alpha^{t}\right) \sigma$ аналогична той, что была в симплектическом случае, с той разницей, что левый верхний угловой 
блок размера $2 \times 2$ имеет вид $\left(\begin{array}{cc}\beta_{2} & * \\ 0 & -\beta_{2}\end{array}\right)$. Это различие носит технический характер и не влияет на результат. Лемма доказана.

Теперь рассмотрим вклад точек $P_{1}, \ldots, P_{N}$. Пусть $\omega_{m}=-\frac{1}{2} \operatorname{res}_{P_{m}} \Omega d z$. Определим $\mathscr{P}_{0}^{D}$ как подпространство в $\mathscr{L}^{D}$, на котором 1 -форма $\delta \kappa d z$ голоморфна. Это то же самое, что множество совместных нулей следующих функций на $\mathscr{L}^{D}$ :

$$
T_{i j l}=\operatorname{res}_{P_{i}^{l}}\left(\left(z-z\left(P_{i}\right)\right)^{j} \kappa d z\right), \quad j=0, \ldots, m_{i}-d_{i},
$$

где $l$ нумерует листы спектральной кривой (рассматриваемой как разветвленное накрытие $\Sigma), d_{i}=\operatorname{ord}_{P_{i}} d z$. Заметим, что слоение, заданное общими поверхностями уровня указанных функций, инвариантно относительно иерархии коммутирующих потоков, так как последние сохраняют спектр $\kappa$ (см. также следующий раздел).

ЛЕмма 6.2. На пространстве $\mathscr{P}_{0}^{D}$

$$
\omega_{m}=\operatorname{res}_{P_{m}} \operatorname{tr}\left(L \Psi^{-1} \delta \Psi \wedge \Psi^{-1} \delta \Psi\right) d z .
$$

ДокАЗАтЕльство. Дифференцируя соотношение $L=\Psi^{-1} K \Psi$, исключим $\delta L$ в определении $\Omega$ и получим

$$
\omega_{m}=\operatorname{res}_{P_{m}} \operatorname{tr}\left(K \delta \Psi \cdot \Psi^{-1} \wedge \delta \Psi \cdot \Psi^{-1}-\delta K \wedge \delta \Psi \cdot \Psi^{-1}\right) d z .
$$

Второе слагаемое голоморфно в $P_{m}$ на пространстве $\mathscr{P}_{0}^{D}$, так как голоморфно выражение $\delta K d z$, а форма $\delta \Psi \cdot \Psi^{-1}$ голоморфна всегда, так как $\delta \Psi$ и $\Psi$ имеют в $P_{m}$ одинаковый порядок (точка $P_{m}$ неподвижна, и ее локальная координата на $\Sigma$ не варьируется, когда мы берем $\delta \Psi)$. В первом слагаемом имеем $\operatorname{tr}(K \delta \Psi$. $\left.\Psi^{-1} \wedge \delta \Psi \cdot \Psi^{-1}\right)=\operatorname{tr}\left(L \Psi^{-1} \delta \Psi \wedge \Psi^{-1} \delta \Psi\right)$. Лемма доказана.

Пусть $\xi$ - касательный вектор к $\mathscr{P}_{0}^{D}$ в точке $L$. Это g-значная мероморфная функция на $\Sigma$, и $\delta \Psi(\xi) \cdot \Psi^{-1}=\xi$. Следовательно, для каждой пары $\xi, \eta$ касательных векторов имеем

$$
\omega_{m}(\xi, \eta)=\operatorname{res}_{P_{m}} \operatorname{tr}(L[\xi, \eta])
$$

Это 2-форма типа Кириллова. Вообще говоря, она вырождена и имеет симплектические листы. В частности, когда $L d z$ имеет простой полюс в $P_{m}$ с вычетом $L_{m}$, выражение $\omega_{m}$ сводится к канонической форме Кириллова на орбите $O_{m}$ элемента $L_{m} \in \mathfrak{g}$. Очевидно, что $(L d z)+D-\mathscr{K} \geqslant 0$ для $L \in \mathscr{L}^{D}$, где $\mathscr{K}$ - дивизор нулей 1-формы $d z$. В отличие от предыдущего случая, если некоторые компоненты дивизора $D-\mathscr{K}$ отрицательны, то соответствующий вклад $\omega_{m}$ обращается в нуль, и $\omega$ очевидным образом вырождается. Мы опускаем детали и ссылаемся в этом месте на полную аналогию с [4].

Так как форма $\omega$ является $G$-инвариантной, то она корректно определена на $\mathscr{P}^{D}=\mathscr{P}_{0}^{D} / G$. Из доказанного в этом разделе вытекает следующее утверждение, совершенно аналогичное теореме 4.1 [4]. 
Tеорема 6.3. Если $D-\mathscr{K} \geqslant 0$, то форма

$$
\omega=\sum_{s}\left(a \delta \kappa_{s} \wedge \delta z_{s}+\delta \alpha_{s}^{t} \wedge \delta \beta_{s}\right)+\sum_{m} \omega_{m}
$$

невырождена на $\mathscr{P}^{D}$. Таким образом, она задает симплектическую струкmypy на $\mathscr{P}^{D}$.

ЗАмечаниЕ 6.4 . В случае $D=\mathscr{K}$ форма $\omega$ невырождена, а вклады точек $P_{m}$ равны нулю. Это соответствует случаю римановых поверхностей без отмеченных точек в подходе Хитчина.

\section{7. Гамильтонова теория}

Следуя [4], мы показываем здесь, что иерархии теоремы 5.2 гамильтоновы для всех классических алгебр Ли, и вычисляем соответствующие гамильтонианы.

Для векторного поля $е$ на $\mathscr{L}^{D}$ определим 1-форму $i_{e} \omega$ соотношением

$$
i_{e} \omega(X)=\omega(e, X)
$$

(где $X$ - произвольное векторное поле). По определению, векторное поле $\partial_{t}-$ гамильтоново, если $i_{\partial_{t}} \omega=\delta H$, где $H$ - некоторая функция, называемая гамильтонианом поля $\partial_{t}$.

Теорема 7.1. Определим векторное поле $\partial_{a}$ соотношением (5.4). Тогда

$$
i_{\partial_{a}} \omega=\delta H_{a},
$$

¿əe

$$
H_{a}=-\frac{1}{k+1} \operatorname{res}_{P_{i}} \operatorname{tr}\left(w^{-m} L^{k+1}\right) d z, \quad a=\left(P_{i}, k, m\right) .
$$

Перед тем как доказывать теорему, рассмотрим некоторые свойства операторов $L, \partial_{a}+M_{a}$ и их спектры. Прежде всего, благодаря уравнению Лакса эти операторы коммутируют, следовательно, строки матрицы $\Psi$ являются собственными векторами для них обоих. Диагональные формы этих двух операторов определяются следующим образом:

$$
K=\Psi L \Psi^{-1}, \quad F_{a}=\Psi\left(\partial_{a}+M_{a}\right) \Psi^{-1} .
$$

Эквивалентно,

$$
\Psi L=K \Psi, \quad \partial_{a} \Psi=\Psi M_{a}-F_{a} \Psi .
$$

Из этих соотношений следует, что

$$
\partial_{a} K=0 .
$$

Как показано выше, $L$ сопряжен матричной функции, голоморфной в $\gamma$-точках, следовательно, его спектр $K$ также голоморфен в этих точках. Но сама матрица $L$ сингулярна и поэтому для $L$ в общем положении $\operatorname{det} \Psi$ имеет (простой) нуль в каждой $\gamma$-точке. Предположим, что

$$
\Psi\left(z-z_{\gamma}\right)=\Psi_{0}+\Psi_{1}\left(z-z_{\gamma}\right)+\cdots, \quad \Psi^{-1}\left(z-z_{\gamma}\right)=\frac{\widetilde{\Psi}_{-1}}{z-z_{\gamma}}+\widetilde{\Psi}_{0}+\cdots
$$


Тогда в общем положении голоморфность $\Psi L$ и соотношение $\Psi \Psi^{-1}=\mathrm{id}$ эквивалентны следующим соотношениям:

$$
\Psi_{0} \alpha=0, \quad \varepsilon \alpha^{t} \sigma \widetilde{\Psi}_{0}=0, \quad \widetilde{\Psi}_{-1}=\alpha \widetilde{\beta}^{t} .
$$

Заметим, что если $\varepsilon=0$ (т.е. $\mathfrak{g}=\mathfrak{g l}(n)$ ), то $\nu=\lambda=0$. Следовательно, и $\nu \alpha^{t} \sigma \widetilde{\Psi}_{0}=\lambda \alpha^{t} \sigma \widetilde{\Psi}_{0}=0$. По той же причине $\varepsilon \alpha^{t} \sigma \alpha=\nu \alpha^{t} \sigma \alpha=\lambda \alpha^{t} \sigma \alpha=0$.

ЗАМЕЧАНИЕ 7.2. В присутствии антиинволюции матрицу $\Psi$ всегда можно выбрать голоморфной в $\gamma$-точках. Действительно, если $\Psi$ имеет полюс в $\gamma$, то подействуем антиинволюцией на первое из соотношений (7.2). Мы получим

$$
\left(\sigma\left(\Psi^{t}\right)^{-1} \sigma^{-1}\right)\left(\sigma L^{t} \sigma^{-1}\right)\left(\sigma \Psi^{t} \sigma^{-1}\right)=\sigma K^{t} \sigma^{-1} .
$$

Заменяя $\sigma L^{t} \sigma^{-1}$ на $-L$, мы получим $\left(\sigma \Psi^{t} \sigma^{-1}\right)^{-1} L\left(\sigma \Psi^{t} \sigma^{-1}\right)=-K^{t}$. В последнем соотношении $-K^{t}$ диагональна, а матрица слева от $L$ голоморфна.

ЗАмЕЧАНИЕ 7.3. Если $L$ имеет полюс второго порядка в $\gamma$, то соотношения $\Psi_{0} \alpha=0$ и $\alpha^{t} \sigma \widetilde{\Psi}_{0}=0$ всегда выполнены в $\gamma$. Действительно, ввиду $\Psi L=K \Psi$ имеем

$$
\left(\Psi_{0}+\Psi_{1}\left(z-z_{\gamma}\right)+\cdots\right)\left(\nu \frac{\alpha \alpha^{t} \sigma}{\left(z-z_{\gamma}\right)^{2}}+\cdots\right)=O(1) .
$$

Следовательно, $\Psi_{0} \alpha \alpha^{t} \sigma=0$. Так как $\alpha \neq 0$ и $\sigma$ невырождена, имеем $\Psi_{0} \alpha=0$. Теперь рассмотрим то же самое соотношение в форме $L \Psi^{-1}=\Psi^{-1} K$. В левой части с помощью вычисления мы получаем $L \Psi^{-1}=\nu \frac{\alpha \alpha^{t} \sigma \widetilde{\Psi}_{0}}{\left(z-z_{\gamma}\right)^{2}}+O\left(\left(z-z_{\gamma}\right)^{-1}\right)$, в то время как правая имеет не более чем простой полюс. Как и выше, заключаем, что $\alpha^{t} \sigma \widetilde{\Psi}_{0}=0$.

ЛЕмма 7.4. При условии выполнения соотношений (7.4) матричные функции $K$ и $F_{a}$ голоморфны во всех $\gamma$-точках.

ДокАЗАТЕЛЬСтво. Имеем:

$$
\begin{gathered}
\Psi L \Psi^{-1}=\frac{\nu \Psi_{0} \alpha \alpha^{t} \sigma \widetilde{\Psi}_{-1}}{\left(z-z_{\gamma}\right)^{3}}+\frac{\nu \Psi_{0} \alpha \alpha^{t} \sigma \widetilde{\Psi}_{0}+\Psi_{0}\left(\alpha \beta^{t}+\varepsilon \beta \alpha^{t}\right) \sigma \widetilde{\Psi}_{-1}+\nu \Psi_{1} \alpha \alpha^{t} \sigma \widetilde{\Psi}_{-1}}{\left(z-z_{\gamma}\right)^{2}} \\
+\frac{\nu \Psi_{0} \alpha \alpha^{t} \sigma \widetilde{\Psi}_{1}+\Psi_{0}\left(\alpha \beta^{t}+\varepsilon \beta \alpha^{t}\right) \sigma \widetilde{\Psi}_{0}+\Psi_{0} L_{0} \widetilde{\Psi}_{-1}+\nu \Psi_{1} \alpha \alpha^{t} \sigma \widetilde{\Psi}_{0}}{z-z_{\gamma}} \\
+\frac{\Psi_{1}\left(\alpha \beta^{t}+\varepsilon \beta \alpha^{t}\right) \sigma \widetilde{\Psi}_{-1}+\nu \Psi_{2} \alpha \alpha t \sigma \widetilde{\Psi}_{-1}}{z-z_{\gamma}}+O(1) .
\end{gathered}
$$

Очевидно, что сингулярная часть этого выражения обращается в нуль при условии (7.4).

Теперь докажем голоморфность спектра $\partial_{a}+M_{a}$. В самом деле, этот спектр представлен матрицей $F_{a}=\Psi\left(\partial_{a}+M_{a}\right) \Psi^{-1}$. Используя разложение для $M_{a}$ и $\Psi^{-1}$ в $\gamma$, получим

$$
\begin{aligned}
\left(\partial_{a}+\right. & \left.M_{a}\right) \Psi^{-1}=\frac{\left(\lambda \alpha \alpha^{t} \sigma\right)\left(\alpha \widetilde{\beta}^{t}\right)}{\left(z-z_{\gamma}\right)^{3}}+\frac{\left(\partial_{a} z_{\gamma}\right) \alpha \widetilde{\beta}^{t}+\lambda \alpha \alpha^{t} \sigma \widetilde{\Psi}_{0}+\left(\alpha \mu_{a}^{t}+\varepsilon \mu_{a} \alpha^{t}\right) \sigma \alpha \widetilde{\beta}^{t}}{\left(z-z_{\gamma}\right)^{2}} \\
& +\frac{\left(\partial_{a} \alpha\right) \widetilde{\beta}^{t}+\alpha\left(\partial_{a} \widetilde{\beta}^{t}\right)+\lambda \alpha \alpha^{t} \sigma \widetilde{\Psi}_{1}+\left(\alpha \mu_{a}^{t}+\varepsilon \mu_{a} \alpha \alpha^{t}\right) \sigma \widetilde{\Psi}_{0}+M_{0 a} \alpha \widetilde{\beta}^{t}}{z-z_{\gamma}}+O(1) .
\end{aligned}
$$


Отбрасывая члены, обращающиеся в нуль ввиду соотношений (7.4) (а также соотношения $\left.\alpha^{t} \sigma \alpha=0\right)$, получим

$$
\left(\partial_{a}+M_{a}\right) \Psi^{-1}=\frac{\left(\partial_{a} z_{\gamma}+\mu_{a}^{t} \sigma \alpha\right) \alpha \widetilde{\beta}^{t}}{\left(z-z_{\gamma}\right)^{2}}+\frac{\left(\partial_{a} \alpha+M_{a 0} \alpha\right) \widetilde{\beta}^{t}}{z-z_{\gamma}}+\frac{\alpha \widehat{\beta}^{t}}{z-z_{\gamma}}+O(1)
$$

где $\widehat{\beta}^{t}=\partial_{a} \widetilde{\beta}^{t}+\lambda \alpha^{t} \sigma \widetilde{\Psi}_{1}+\mu_{a}^{t} \sigma \Psi_{0}$. Ввиду (4.3) первое слагаемое обращает-

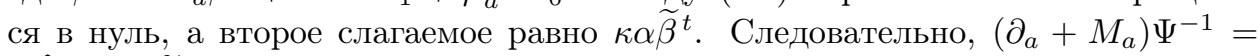
$\frac{\alpha \widehat{\beta}^{t}+\kappa \alpha \widetilde{\beta}^{t}}{z-z_{\gamma}}+O(1)$ и $\Psi\left(\partial_{a}+M_{a}\right) \Psi^{-1}=O(1)$, поскольку $\Psi_{0} \alpha=0$. Лемма доказана.

ДоКАЗАТЕЛЬСТво теОРЕмы 7.1. Коль скоро доказана лемма 7.4, оставшаяся часть доказательства теоремы такова же, как в [4]. Мы приведем его здесь для полноты.

По определению,

$$
i_{\partial_{a}} \omega=\omega\left(\partial_{a}, \cdot\right)=-\frac{1}{2}\left(\sum_{s=1}^{K} \operatorname{res}_{\gamma_{s}} \Lambda+\sum_{i=1}^{N} \operatorname{res}_{P_{i}} \Lambda\right),
$$

где $\Lambda=\Omega\left(\partial_{a}, \cdot\right)$. Ввиду соотношений $\delta \Psi\left(\partial_{a}\right)=\partial_{a} \Psi$ и $\delta L\left(\partial_{a}\right)=\partial_{a} L$ (значение дифференциала на векторном поле равно производной вдоль этого векторного поля) имеем

$$
\Lambda=\operatorname{tr}\left(\partial_{a} \Psi \cdot \delta L \cdot \Psi^{-1}-\delta \Psi \cdot \partial_{a} L \cdot \Psi^{-1}-\partial_{a} K \cdot \delta \Psi \cdot \Psi^{-1}+\delta K \cdot \partial_{a} \Psi \cdot \Psi^{-1}\right) .
$$

Ввиду (7.2) и уравнений Лакса,

$$
\begin{aligned}
\Lambda & =\operatorname{tr}\left(\left(\Psi M_{a}-F_{a} \Psi\right) \delta L \cdot \Psi^{-1}-\delta \Psi\left[L, M_{a}\right] \Psi^{-1}+\delta K\left(\Psi M_{a}-F_{a} \Psi\right) \Psi^{-1}\right) \\
& =\operatorname{tr}\left(M_{a} \delta L-F_{a} \Psi \delta L \cdot \Psi^{-1}-\delta \Psi\left[L, M_{a}\right] \Psi^{-1}+\delta K \Psi M_{a} \Psi^{-1}-\delta K F_{a}\right) .
\end{aligned}
$$

Преобразуем средний член. Согласно $\Psi L=K \Psi$ имеем $\delta \Psi \cdot L=-\Psi \delta L+\delta K \Psi+$ $K \delta \Psi$. Следовательно,

$$
\begin{aligned}
\operatorname{tr} \delta \Psi\left[L, M_{a}\right] \Psi^{-1}= & \operatorname{tr}\left((\delta \Psi \cdot L) M_{a} \Psi^{-1}-\delta \Psi M_{a} L \Psi^{-1}\right) \\
= & \operatorname{tr}\left((-\Psi \delta L+\delta K \Psi+K \delta \Psi) M_{a} \Psi^{-1}-\delta \Psi M_{a} L \Psi^{-1}\right) \\
= & \operatorname{tr}\left(-\Psi \delta L M_{a} \Psi^{-1}+\delta K \Psi M_{a} \Psi^{-1}\right. \\
& \left.\quad+K \delta \Psi M_{a} \Psi^{-1}-\delta \Psi M_{a} L \Psi^{-1}\right) .
\end{aligned}
$$

Последние два члена уничтожаются, так как

$$
\operatorname{tr}\left(\delta \Psi M_{a} L \Psi^{-1}\right)=\operatorname{tr}\left(\delta \Psi M_{a} \Psi^{-1}\left(\Psi L \Psi^{-1}\right)\right)=\operatorname{tr}\left(\delta \Psi M_{a} \Psi^{-1} K\right),
$$

и мы получаем

$$
\operatorname{tr} \delta \Psi\left[L, M_{a}\right] \Psi^{-1}=\operatorname{tr}\left(-\delta L M_{a}+\delta K \Psi M_{a} \Psi^{-1}\right) .
$$

Подставляя это в последнее выражение для $\Lambda$, находим, что

$$
\Lambda=\operatorname{tr}\left(2 M_{a} \delta L-F_{a} \Psi \delta L \cdot \Psi^{-1}-\delta K F_{a}\right) .
$$


Последние два члена здесь равны под знаком следа, что может быть показано заменой $\Psi \delta L$ на $-\delta \Psi L+\delta K \Psi+K \delta \Psi$. Окончательно мы получаем

$$
\Lambda=\operatorname{tr}\left(2 M_{a} \delta L-2 \delta K F_{a}\right) .
$$

Это дает

$$
i_{\partial_{a}} \omega=\sum_{j=1}^{N} \operatorname{res}_{P_{j}} \operatorname{tr}\left(\delta K F_{a}\right) d z-R_{a}
$$

где

$$
R_{a}=\sum_{s=1}^{K} \operatorname{res}_{\gamma_{s}} \operatorname{tr}\left(\delta L M_{a}\right) d z+\sum_{j=1}^{N} \operatorname{res}_{P_{j}} \operatorname{tr}\left(\delta L M_{a}\right) d z .
$$

Заметим, что сумма по $\gamma$-точкам в $(7.5)$ обращается в нуль, поскольку $\delta K$ и $F_{a}$ голоморфны в $\gamma$-точках (лемма 7.4). Заметим также, что $\gamma$-точки и точки $P_{j}-$ это не все сингулярности функции $F_{a}$. В самом деле, $F_{a}=-\partial_{a} \Psi \cdot \Psi^{-1}-$ $\Psi M_{a} \Psi^{-1}$, а $\Psi^{-1}$ имеет полюсы в точках ветвления собственных значений $L$.

С другой стороны, $L$ и $M_{a}$ голоморфны везде за исключением точек $\gamma_{s}$ и $P_{j}$. По этой причине все особенности 1 -формы $M_{a} \delta L$ находятся в этих точках. Следовательно, $R_{a}=0$, так как это сумма вычетов мероморфной 1-формы по всем ее полюсам. Более того, по построению $M_{a}$ матрица $F_{a}$ голоморфна в $P_{j}, j \neq i$, для всех времен $a$, соответствующих точке $P_{i}$. Следовательно, для таких времен

$$
i_{\partial_{a}} \omega=\operatorname{res}_{P_{i}} \operatorname{tr}\left(\delta K F_{a}\right) d z .
$$

Имеем $F_{a}=-\partial_{a} \Psi \cdot \Psi^{-1}-\Psi M_{a} \Psi^{-1}$, и $\partial_{a} \Psi \cdot \Psi^{-1}$ голоморфно в $P_{i}$ ввиду независимости координаты $P_{i}$ от времен, следовательно, $\partial_{a} \Psi$ и $\Psi$ имеют один и тот же порядок в $P_{i}$. Таким образом, $F_{a}=-\Psi M_{a} \Psi^{-1}+O(1)$. По определению, $M_{a}=w^{-m} L^{k}+O(1)$ в точке $P_{i}$ для $a=\left(P_{i}, k, m\right)$. Отсюда следует, что

$$
\Psi M_{a} \Psi^{-1}=w^{-m} \Psi L^{k} \Psi^{-1}+O(1),
$$

так как $\Psi$ голоморфно обратима в $P_{i}$. Но $\Psi L^{k} \Psi^{-1}=K^{k}$, следовательно, $F_{a}=$ $w^{-m} K^{k}+O(1)$ в $P_{i}$. Так как $\delta K d z$ голоморфно в $P_{i}$, получим

$$
\begin{aligned}
i_{\partial_{a}} \omega=-\operatorname{res}_{P_{i}} \operatorname{tr}\left(w_{i}^{-m} \delta K \cdot K^{k}\right) d z & =-\frac{1}{k+1} \operatorname{res}_{P_{i}} \delta \operatorname{tr}\left(w_{i}^{-m} K^{k+1}\right) \\
& =-\frac{1}{k+1} \delta \operatorname{res}_{P_{i}} \operatorname{tr}\left(w_{i}^{-m} L^{k+1}\right)=\delta H_{a} .
\end{aligned}
$$

Теорема доказана.

Гамильтонианы $H_{a}$ находятся в инволюции, так как они зависят только от спектральных параметров. За деталями мы отсылаем к [4].

\section{8. Примеры: системы Калоджеро-Мозера}

Начнем с примера, рассмотренного в [4] - эллиптической модели Калоджеро-Мозера для $\mathfrak{g}=\mathfrak{g l}(n)$. Определим оператор Лакса следующим образом:

$$
L_{i j}=f_{i j} \frac{\sigma\left(z+q_{j}-q_{i}\right) \sigma\left(z-q_{j}\right) \sigma\left(q_{i}\right)}{\sigma(z) \sigma\left(z-q_{i}\right) \sigma\left(q_{j}-q_{i}\right) \sigma\left(q_{j}\right)} \quad(i \neq j), \quad L_{j j}=p_{j},
$$


где $f_{i j} \in \mathbb{C}$ - постоянные, $\sigma$ - функция Вейерштрасса. С точностью до констант $f_{i j}$ такая форма $L$ предопределена требованиями, что $L$ - эллиптическая функция и что она имеет простые полюсы в точках $z=q_{i}(i=1, \ldots, n)$ и $z=0$. Точка $z=0$ является единственной неподвижной точкой. Путем редукции остающейся калибровочной свободы в [4] получено, что $f_{i j} f_{j i}=1$. Для гамильтониана второго порядка мы имеем согласно теореме 7.1, с точностью до нормировки,

$$
H=\operatorname{res}_{z=0} z^{-1}\left(-\frac{1}{2} \sum_{j=1}^{n} p_{j}^{2}-\sum_{i<j} L_{i j} L_{j i}\right) .
$$

По теореме сложения для функций Вейерштрасса

$$
-L_{i j} L_{j i}=\frac{\sigma\left(z+q_{i}-q_{j}\right) \sigma\left(z+q_{j}-q_{i}\right)}{\sigma(z)^{2} \sigma\left(q_{i}-q_{j}\right)^{2}}=\wp\left(q_{i}-q_{j}\right)-\wp(z),
$$

следовательно,

$$
H=-\frac{1}{2} \sum_{j=1}^{n} p_{j}^{2}+\sum_{i<j} \wp\left(q_{i}-q_{j}\right) .
$$

Теперь рассмотрим случай $\mathfrak{s o}(2 n)$. Мы продемонстрируем здесь другой метод построения иерархии для эллиптических кривых. Пусть $M$-операторы принимают значения в той же алгебре, т. е. произвольный $M$-оператор имеет вид

$$
M=\left(\begin{array}{cc}
A & B \\
C & -A^{t}
\end{array}\right), \quad B^{t}=-B, \quad C^{t}=-C .
$$

Мы предполагаем, что

$$
\begin{gathered}
K=2 n, \\
(A)+D+\sum_{i=1}^{n} q_{i} \geqslant 0, \quad(C)+D+\sum_{i=1}^{n} q_{i} \geqslant 0, \quad(B)+D+\sum_{i=1}^{n}\left(-q_{i}\right) \geqslant 0,
\end{gathered}
$$

где $D=\sum m_{i} P_{i}$ как и раньше. Следовательно, подматрицы $A, C$ голоморфны в точках $-q_{i}$, а подматрица $B-$ в точках $q_{i}$. Обозначим пространство таких $M$-операторов снова $\mathscr{N}^{D}$.

Лемма 8.1. $\operatorname{dim} \mathscr{N}^{D}=(\operatorname{dim} \mathfrak{g})(\operatorname{deg} D+1)$.

ДокАЗАтЕльСтво. Обозначим размерность подпространства элементов в $\mathfrak{g}$ таких, что $B=0$, через $d^{\prime}$, а подпространство элементов таких, что $A=C=0$, через $d^{\prime \prime}$. Тогда $\operatorname{dim} \mathfrak{g}=d^{\prime}+d^{\prime \prime}$. По теореме Римана-Роха для $g=1$ получаем

$$
\operatorname{dim} \mathscr{N}^{D}=d^{\prime}(\operatorname{deg} D+n)+d^{\prime \prime}(\operatorname{deg} D+n)-\left(d^{\prime}-n\right) n-\left(d^{\prime \prime}-n\right) n-n .
$$

Последние три слагаемых отвечают соотношениям. В точке $q_{i}$ у нас $d^{\prime}$ соотношений $\operatorname{res}_{q_{i}}\left(\begin{array}{cc}A & 0 \\ C & -A^{t}\end{array}\right)=\left(\begin{array}{c}\alpha_{i}^{\prime} \\ \alpha_{i}^{\prime \prime}\end{array}\right)\left(\mu_{i}^{\prime t}, \mu_{i}^{\prime \prime t}\right) \sigma-\left(\begin{array}{c}\mu_{i}^{\prime} \\ \mu_{i}^{\prime \prime}\end{array}\right)\left(\alpha_{i}^{\prime t}, \alpha_{i}^{\prime \prime t}\right) \sigma$, где $\sigma=\left(\begin{array}{cc}0 & E \\ E & 0\end{array}\right)$, $E$ - единичная матрица. Мы также имеем $2 n$ параметров $\mu_{i}^{\prime}, \mu_{i}^{\prime \prime}$, удовлетворяющих $n$ соотношениям $\alpha_{i}^{\prime} \mu_{i}^{\prime t}-\mu_{i}^{\prime} \alpha_{i}^{\prime t}=0$, которые эквивалентны обращению в нуль $B$-блока. 
В точке $-q_{i}$ мы имеем $d^{\prime \prime}$ соотношений $\operatorname{res}_{-q_{i}} B=\left(\alpha_{-i} \mu_{-i}^{t}-\mu_{-i} \alpha_{-i}^{t}\right) \sigma$, где $\alpha_{-i}=\left(\begin{array}{c}\alpha_{-i}^{\prime} \\ 0\end{array}\right), \mu_{-i}=\left(\begin{array}{c}\mu_{-i}^{\prime} \\ 0\end{array}\right)$, и $n$ свободных параметров, идущих от $\mu_{-i}^{\prime}$.

Последние $n$ соотношений возникают благодаря тому, что движения точек $q_{i}$ и $-q_{i}$ связаны между собой. Так как $\dot{q}_{i}=-\mu_{i}^{\prime t} \alpha_{i}^{\prime \prime}-\mu_{i}^{\prime \prime t} \alpha_{i}^{\prime},-\dot{q}_{i}=-\mu_{-i}^{\prime t} \alpha_{-i}^{\prime}$ и $\dot{q}_{i}+\left(-\dot{q}_{i}\right)=0$, мы получаем эти дополнительные $n$ соотношений.

Окончательно,

$$
\operatorname{dim} \mathscr{N}^{D}=(\operatorname{dim} \mathfrak{g}) \operatorname{deg} D+\left(2 n^{2}-n\right)=(\operatorname{dim} \mathfrak{g})(\operatorname{deg} D+1) .
$$

Лемма доказана.

Мы берем $L$ в той же форме, что и $M$, где $A$ имеет вид (8.1). При $i<j$ мы берем

$$
B_{i j}=f_{i j}^{B} \frac{\sigma\left(z+q_{j}+q_{i}\right) \sigma\left(z-q_{j}\right)}{\sigma(z) \sigma\left(z+q_{i}\right) \sigma\left(q_{i}+q_{j}\right)}, \quad C_{j i}=f_{j i}^{C} \frac{\sigma\left(z-q_{j}-q_{i}\right) \sigma\left(z+q_{i}\right)}{\sigma(z) \sigma\left(z-q_{j}\right) \sigma\left(q_{i}+q_{j}\right)},
$$

где $f_{i j}^{B}, f_{i j}^{C} \in \mathbb{C}$ - постоянные. Эти соотношения определяют матрицы $B$ и $C$ ввиду их кососимметричности. Аналогично случаю $\mathfrak{g l}(n)$ путем редукции остающейся калибровочной свободы мы получаем $f_{i j}^{B} f_{j i}^{C}=-1$ (при этом используется соотношение $\alpha^{t} \sigma \alpha=0$, которое сводится в данном случае к $\left.\alpha^{\prime t} \alpha^{\prime \prime}=0\right)$. Для гамильтониана мы имеем

$$
\begin{aligned}
H & =-\operatorname{res}_{z=0} z^{-1}\left(\sum_{i=1}^{n} p_{i}^{2}+2 \sum_{i<j} A_{i j} A_{j i}+2 \sum_{i<j} B_{i j} C_{j i}\right) \\
& =-\sum_{i=1}^{n} p_{i}^{2}+2 \sum_{i<j} \wp\left(q_{i}-q_{j}\right)+2 \sum_{i<j} \wp\left(q_{i}+q_{j}\right) .
\end{aligned}
$$

ЗАмЕчАНИЕ 8.2. Имеется простое соотношение между нашим оператором Лакса и оператором Лакса, данным в [12]. Для $\mathfrak{g}=\mathfrak{s o}(n)$ последний имеет вид

$$
\widetilde{L}=\left(\begin{array}{cc}
P & 0 \\
0 & -P
\end{array}\right)+\left(\begin{array}{cc}
A & B \\
C & A^{t}
\end{array}\right), \quad B^{t}=B, \quad C^{t}=C,
$$

где $P=\operatorname{diag}\left(p_{1}, \ldots, p_{n}\right)$ и $A, B, C$ - матрицы с нулевой диагональю, остальные элементы которых являются функциями Бейкера-Ахиезера на эллиптической кривой. В этих терминах наш оператор Лакса имеет вид

$$
L=\left(\begin{array}{cc}
P & 0 \\
0 & -P
\end{array}\right)+\left(\begin{array}{cc}
A & B \\
C & -A^{t}
\end{array}\right), \quad B^{t}=-B, \quad C^{t}=-C .
$$

Оператор $L$ переводится в $\widetilde{L}$ следующим преобразованием:

$$
P \rightarrow P, \quad A \rightarrow A, \quad B=b-b^{t} \rightarrow B^{\prime}=b+b^{t}, \quad C=c-c^{t} \rightarrow C^{\prime}=-\left(c+c^{t}\right),
$$

где $b$ и $c$ - верхнетреугольные матрицы. Например, для $\mathfrak{g}=s o(4)$ оно выглядит так:

$$
\left(\begin{array}{cccc}
p_{1} & a_{12} & 0 & b \\
a_{21} & p_{2} & -b & 0 \\
0 & c & -p_{1} & -a_{21} \\
-c & 0 & -a_{12} & -p_{2}
\end{array}\right) \rightarrow\left(\begin{array}{cccc}
p_{1} & a_{12} & 0 & b \\
a_{21} & p_{2} & b & 0 \\
0 & -c & -p_{1} & a_{21} \\
-c & 0 & a_{12} & -p_{2}
\end{array}\right)
$$

где $b, c \in \mathbb{C}$. Это преобразование сохраняет $\operatorname{tr} L^{2}, \operatorname{tr} L^{3}=0$ и $\operatorname{tr} L^{4}$. 
Если элементы матрицы $L$ даны формулами (8.4) и (8.1), то композиция указанного преобразования и сопряжения диагональной матрицей $e^{q \zeta(z)}$ (где $\zeta-$ $\zeta$-функция Вейерштрасса, $\left.q=\operatorname{diag}\left(q_{1}, \ldots, q_{n},-q_{1}, \ldots,-q_{n}\right)\right)$ приводит к оператору Лакса из [12].

Имеется аналогичная связь между двумя операторами Лакса и в случае остальных классических алгебр Ли $\mathfrak{g}$. Для $\mathfrak{g}=\mathfrak{g l}(n)$ калибровочная эквивалентность $L$ с известным оператором Лакса [11] отмечена в [4].

Теперь рассмотрим случай $\mathfrak{g}=\mathfrak{s p}(2 n)$. Определим $\mathscr{N}^{D}$ как пространство $M$-операторов, принимающих значения в $\mathfrak{g}^{\diamond}=\mathfrak{t} \mathfrak{s p}(2 n)$ и имеющих $n+1$ пару двойных полюсов $\pm q_{i}(i=1, \ldots, n+1)$. Таким образом,

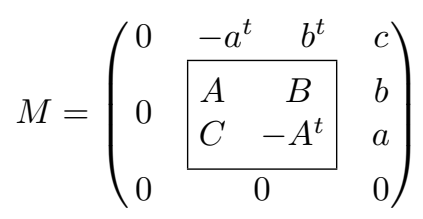

где $a, b \in \mathbb{C}^{n}, c \in \mathbb{C}, A, B, C$ - матрицы размера $n \times n, B=B^{t}, C=C^{t}$. Мы предполагаем, что $(A)+D+\sum_{i=1}^{n+1} 2 q_{i} \geqslant 0$ (и то же самое для дивизоров матричных функций $C, a, b, c)$ и $(B)+D+\sum_{i=1}^{n+1} 2\left(-q_{i}\right) \geqslant 0$.

Аналог соотношения (8.3) записывается в виде

$$
\begin{aligned}
\operatorname{dim} \mathscr{N}^{D}=d^{\prime} & (\operatorname{deg} D+2 n+2)+d^{\prime \prime}(\operatorname{deg} D+2 n+2) \\
& -\left(2 d^{\prime}-n-1\right)(n+1)-\left(2 d^{\prime \prime}+1-n\right)(n+1)-n,
\end{aligned}
$$

где $d^{\prime \prime}$ - размерность $B$-блока, а $d^{\prime}$ - размерность оставшейся части матрицы. Для объяснения второй строки формулы заметим, что в каждом полюсе $q_{1}, \ldots, q_{n}$ у нас имеется $2 d^{\prime}$ соотношений, соответствующих тому, что вид членов порядков -1 и -2 предписан. Мы также хотим иметь соотношение $\nu=0$, что ведет к еще одному соотношению, но оно компенсируется тем, что мы на самом деле не заботимся об асимптотическом поведении элемента $c$ матрицы $M$, так как он отвечает центру алгебры $\mathfrak{t} \mathfrak{p}(2 n)$, так что мы можем опустить соотношение на полюс второго порядка для этого элемента. Мы также имеем $2 n+1$ параметров, идущих от $\mu$, подчиненных $n$ условиям обращения в нуль $\operatorname{res}_{q_{i}} B$. Таким образом, мы имеем $2 d^{\prime}-(n+1)$ эффективных соотношений для каждого $q_{i}$. Отметим также, что параметры $\lambda$ компенсируются соотношениями $\alpha^{t} \sigma M_{1} \alpha=0$. В точке $-q_{i}$ имеется $2 d^{\prime \prime}+1-n$ соотношений, так как $\mu$ для блока $B$ имеет размерность $n$, и добавочное соотношение для $\nu$ ничем не компенсировано. Последнее $(-n)$ соответствует соотношениям $\dot{q}_{i}+\left(-\dot{q}_{i}\right)=0$, $i=1, \ldots, n$ (и мы не заботимся о поведении последней пары полюсов).

Таким образом, мы получаем

$$
\operatorname{dim} \mathscr{N}^{D}=\left(\operatorname{dim} \mathfrak{g}^{\diamond}\right)(\operatorname{deg} D)+2 n^{2}+n .
$$

Заметим, что $2 n^{2}+n=\operatorname{dim} \mathfrak{g}$, т. е. это есть размерность подматрицы $\left(\begin{array}{cc}A & B \\ C & -A^{t}\end{array}\right)$ матрицы $M$. Таким образом, в конструкции операторов $M_{a}$ вместо условия 
нормировки (iii) (с. 165) мы можем потребовать, чтобы в $P_{0}$ обращалась в нуль эта подматрица.

Возьмем $L$ в том же виде, что и $M$, где соответствующие элементы $A_{i j}$, $B_{i j}, C_{i j}$ определены формулами (8.1) (с $A$ вместо $\left.L\right)$, (8.4). Формулы (8.4) имеют смысл для $i=j$, и вклад соответствующих элементов в гамильтониан второго порядка равен

$$
B_{i i} C_{i i}=f_{i i}^{B} f_{i i}^{C}\left(\wp\left(2 q_{i}\right)-\wp(z)\right),
$$

где можно положить $f_{i i}^{B} f_{i i}^{C}$ равным постоянному $\varepsilon$. Подматрицы $a$ и $b$ матрицы $L$ не дают вклада в гамильтониан вне зависимости от их конкретного вида. Следовательно,

$$
H=-\sum_{i=1}^{n} p_{i}^{2}+2 \sum_{i<j} \wp\left(q_{i}-q_{j}\right)+2 \sum_{i<j} \wp\left(q_{i}+q_{j}\right)+\varepsilon \sum_{i=1}^{n} \wp\left(2 q_{i}\right),
$$

что является общеизвестной формой гамильтониана эллиптической модели Калоджеро-Мозера в симплектическом случае.

\section{Список литературы}

[1] И. М. Кричевер, С.П. Новиков, "Голоморфные расслоения над алгебраическими кривыми и нелинейные уравнения", УМН, 35:6 (1980), 47-68; англ. пер.: I. M. Krichever, S.P. Novikov, "Holomorphic bundles on algebraic curves and nonlinear equations", Russian Math. Surveys, 35:6 (1980), 53-79.

[2] И. М. Кричевер, С. П. Новиков, "Голоморфные расслоения над римановыми поверхностями и уравнение Кадомцева-Петвиашвили (КП). I", Функи. анализ и его прил., 12:4 (1978), 41-52; англ. пер.: I. M. Krichever, S. P. Novikov, "Holomorphic bundles over Riemann surfaces and the Kadomtsev-Petviashvili equation. I", Funct. Anal. Appl., 12:4 (1978), 276-286.

[3] И. М. Кричевер, "Коммутативные кольца обыкновенных линейных дифференциальных операторов", Функи. анализ и его прил., 12:3 (1978), 20-31; англ. пер.: I. M. Krichever, "Commutative rings of ordinary linear differential operators", Funct. Anal. Appl., 12:3 (1978), 175-185.

[4] I. M. Krichever, "Vector bundles and Lax equations on algebraic curves", Comm. Math. Phys., 229:2 (2002), 229-269.

[5] И. М. Кричевер, О.К. Шейнман, “Алгебры операторов Лакса”, Функи. анализ и его прил., 41:4 (2007), 46-59; англ. пер.: I. M. Krichever, O. K. Sheĭnman, "Lax operator algebras", Funct. Anal. Appl., 41:4 (2007), 284-294; arXiv: math/0701648.

[6] O.K. Sheinman, "On certain current algebras related to finite-zone integration", Geometry, topology, and mathematical physics, Amer. Math. Soc. Transl. Ser. 2, 224, eds. V. M. Buchstaber, I. M. Krichever, Amer. Math. Soc., Providence, RI, 2008, $271-284$.

[7] О.К. Шейнман, “Алгебры операторов Лакса и интегрируемые иерархии”, Геометрия, топология и математическая физика. I, Сб. статей. К 70-летию со дня рождения академика Сергея Петровича Новикова, Тр. МИАН, 263, МАИК, М., 2008, 216-226; англ. пер.: O. K. Sheinman, "Lax operator algebras and integrable hierarchies", Proc. Steklov Inst. Math., 263 (2008), 204-213.

[8] А.Н. Тюрин, "Классификация векторных расслоений над алгебраической кривой произвольного рода", Изв. АН СССР. Сер. матем., 29:3 (1965), 657-688. 
[9] O.K. Sheinman, "Affine Krichever-Novikov algebras, their representations and applications", Geometry, topology, and mathematical physics, S. P. Novikov's seminar (Moscow, 2002-2003), Amer. Math. Soc. Transl. Ser. 2, 212, Amer. Math. Soc., Providence, RI, 2004, 297-316; arXiv: math/0304020.

[10] М. Шлихенмайер, О.К. Шейнман, “Центральные расширения алгебр операторов Лакса", УМН, 63:4 (2008), 131-172; англ. пер.: M. Schlichenmaier, O. K. Sheinman, "Central extensions of Lax operator algebras", Russian Math. Surveys, 63:4 (2008), $727-766$.

[11] И. М. Кричевер, “Эллиптические решения уравнения Кадомцева-Петвиашвили и интегрируемые системы частиц", Функи. анализ и его прил., 14:4 (1980), 45-54; I. M. Krichever, "Elliptic solutions of the Kadomtsev-Petviashvili equation and integrable systems of particles", Funct. Anal. Appl., 14:4 (1980), 282-290.

[12] E. D'Hocker, D. H. Phong, Calogero-Moser Lax pairs with spectral parameter for general Lie algebras, arXiv: hep-th/9804124.

[13] И. М. Кричевер, С.П. Новиков, “Алгебры типа Вирасоро, римановы поверхности и структуры теории солитонов”, Функи. анализ и его прил., 21:2 (1987), 46-63; англ. пер.: I. M. Krichever, S. P. Novikov, "Algebras of Virasoro type, Riemann surfaces and structures of the theory of solitons", Funct. Anal. Appl., 21:2 (1987), $126-142$.

О. К. Шейнман (О.К. Sheinman)

Математический институт им. В. А. Стеклова РАН;

Независимый московский университет

E-mail: sheinman@mi.ras.ru
Поступила в редакцию

09.12 .2010 